\title{
Quantum dynamics on (super)groups: Constraints and the "BRST" supergroup
}

\author{
V. Aldaya ${ }^{\text {a) }}$ and R. Loll \\ The Blackett Laboratory, Imperial College, London SW7 2BZ, England \\ J. Navarro-Salas \\ Departamento de Fîsica Teórica, Facultad de Fisicas, Universidad de Valencia, Burjasot (Valencia), Spain, \\ and Centro Mixto Universidad de Valencia, C.S.I.C., Valencia, Spain
}

(Received 6 April 1990; accepted for publication 15 August 1990)

\begin{abstract}
The smallest supergroup $\widetilde{\mathfrak{B}}(K)$ containing among its generators those of a constraint group $K$, the BRST charge, and the corresponding ghost and antighost operators is constructed. The supergroup $\widetilde{\mathfrak{B}}(K)$ can be enlarged to include the ordinary dynamical variables of the unconstrained physical system. In a group approach to quantization $\overline{\mathfrak{B}}(K)$ generalizes the ordinary $U(1)$ phase invariance of wave functions. In particular this mechanism reproduces the essential features of the BRST quantization. When $K$ is diff $S^{1}$ the critical values of string theory are those for which the space of polarized functions on the enlarged BRST supergroup is not irreducible.
\end{abstract}

\section{INTRODUCTION}

This paper is devoted to the general construction and study of a supergroup $\widetilde{\mathfrak{B}}(K)$ introduced previously, ${ }^{1}$ containing among its generators those of a constraint subgroup $K$, the BRST charge and the corresponding ghost and antighost operators. This supergroup will constitute a crucial ingredient in the framework of a group approach to quantization $(\mathrm{GAQ})^{2-4}$ to be considered. In fact, $\mathfrak{B}(K)$ generalizes the ordinary $U$ (1) subgroup of the Heisenberg-Weyllikc quantum symmetry, i.e., the phase symmetry of the wave functions. From a mathematical point of view $\widetilde{\mathfrak{B}}(K)$ is the fiber of a bigger supergroup, including general coordinates and momenta, with a principal bundle structure. As a by-product we shall recover the well-known BRST quantization. ${ }^{5-9}$ In particular, the group $\widetilde{\mathfrak{B}}$ (diff $S^{1}$ ) will be enlarged to a group $\widetilde{B}$ (diff $S^{1}$, diff $S^{1}\left(S \mathscr{L} \mathbb{R}^{1, d-1}\right.$ ) which includes the phase space operators $\alpha_{n}^{\mu}$ for open strings, ${ }^{10}$ i.e., the loop space group $\mathscr{L} \mathbb{R}^{1, d-1}$. Since the condition $Q^{2}=\frac{1}{2}\{Q, Q\}=0$ (Ref. 11 ) is trivially satisfied in the new superalgebra, the critical dimension of string theory will emerge in a nonconventional way. In Ref. 4 it was proved that the space of polarized functions on the Virasoro group is not, in general, irreducible. The irreducible Hilbert space was obtained by just taking the invariant subspace defined by the orbit of the enveloping algebra through the vacuum. This space is a proper subspace for those (critical) values of $c$ and $h$ for which the Kac determinant vanishes. In the present case, the critical values of string theory will arise in a completely similar way. The nonirreducibility of the space of polarized functions is the group-theoretical translation of the $Q^{2}$ anomaly.

The use of a group as the essential structure in the quantization of a physical problem has found application in many areas. Apart from the natural application in the representa-

\footnotetext{
a) Present address: IFIC, Centro Mixto Universidad de Valencia, C.S.I.C. and the Departamento de Física Teórica de la Universidad de Granada, Granada, Spain.
}

tion theory of (infinite-dimensional) Lie groups ${ }^{12}$ it has been successful in the treatment of nonlinear problems in general and in those usually analyzed by means of the inverse scattering method in particular. Yet for very special cases that should have been of trivial significance, but where the standard geometric quantization methods ${ }^{13}$ are fraught with difficulties, group approaches show up their skill. This is the case, for instance, for the quantization of a free-particle constrained to a circle that is required to describe the dynamics of strings moving on a torus, ${ }^{14}$ or the quantization of identical particles. ${ }^{3}$ It has also succeeded in quantizing gravitational models with basic coordinates obeying (nonstandard) affine Poisson brackets. ${ }^{15}$ It is needless to reiterate the clarifying role of a group approach to quantization in relating symmetry and quantization, dealing with the classical limit, position operators, etc. ${ }^{2}$

The group theoretic approach to quantization represents an improvement of the standard methods in many respects. Of particular significance is the fact that the prescription for quantizing physical quantities is neatly stated in algebraic terms irrespective of the actual form of the basic commutators of the theory, which is very important for nonlinear problems. Another example is the algebraic formulation of the normal ordering. Once a particular subgroup (denominated polarization subgroup, see Sec. II) is chosen, the physical operators do automatically appear in normal ordered form. This avoids additional regularization procedures and sometimes true infinities, ${ }^{16}$ i.e., infinities which seem to be unavoidable from the point of view of the standard quantization techniques. Moreover, the group structure provides an adequate framework for incorporating different sorts of constraints which now appear as well-defined subgroups. Even when no truly symplectic form can be defined as a consequence of the constraints, the Lie product still provides us with a (generalized) Poisson bracket.

Finally, it must be pointed out that the important structure in this approach to quantization is the basic associative law supporting a Lie group, a Lie supergroup or, even more, 
just an algebraic group, rather than the concrete differentiable Lie structure. Indeed the quantization techniques based on deformation theory of Lie algebras ${ }^{17,18}$ finish with a nonLie associative algebra. ${ }^{19}$ In the case where the starting point is just an algebraic group there also appears the possibility of considering discrete symmetry transformations that play an essential role in the quantum dynamics ${ }^{14,3}$ or, more general$1 y$, formal group laws in terms of noncontinuous functions or even distributions.

This paper is organized as follows. In Sec. II we reexamine the essential ingredients of a specific group approach to quantization (GAQ) also particularly suited for incorporating constraints. We show how the (irreducible) Fock space arises from the space of polarized functions on the group. Section III is devoted to illustrating the GAQ method by means of the very simple nontrivial example of the free-particle constrained by the (non-Abelian) rotation group. In Sec. IV we seek a supergroup $\widetilde{\mathfrak{B}}(K)$ containing an arbitrary constraint group $K$, the BRST fermionic operator $Q$, the ghost and antighost operators, as well as some additional bosonic operators that are needed to close the supergroup. The general set of representations of $\widetilde{\mathfrak{B}}(K), \operatorname{dim} K<\infty$, is given, although the mechanism equally well applies in $\operatorname{dim} K=\infty$. In Sec. V two examples, one for $K=\mathrm{SU}(2)$ and another for $K=\operatorname{diff} S^{1}$, illustrate the previous section. The second example shows some subtleties arising in infinite dimensions and the capability of GAQ to handle such systems. Finally, in Sec. VI we account for the by now almost standard BRST construction $^{5}$ from our group point of view. It requires enlarging $\widetilde{\mathfrak{B}}(K)$ so as to incorporate the ordinary coordinates and momenta of the particular physical system under study. Of course the structure of the enlarged supergroup depends upon the algebraic structure of the unconstrained system. The constrained Galilean particle and the bosonic string will be analyzed. The second example illustrates the connection between the nonirreducibility of the space of polarized wave functions (for $d=26, c^{\prime}=24$ ) and the presence of the (Virasoro) anomaly of the theory.

\section{DYNAMICS ON A GROUP AND CONSTRAINTS}

We shall be concerned here with a concrete group approach to quantization (GAQ) which has been developed over the last few years ${ }^{24}$ and which shares many basic features with other more or less recent group theoretic quantization formalisms. ${ }^{14,18,19}$ The essential idea of $G \Lambda Q$ is to replace the Poisson brackets (or a deformed version of them) for the basic quantities characterizing a concrete physical system, for example, $x^{i}, p_{j}$, and $H$, with the Lie bracket of a Lie algebra with as many additional parameters as needed to close the algebra. One notices at once that at least one new generator must be added to the pair $x^{i}, p_{j}$, since the Poisson bracket

$$
\left\{x^{i}, p_{j}\right\}=\delta_{j}^{i}, \quad i, j=1, \ldots, n
$$

can only be thought of as a Lie bracket of a Lie algebra if the additional generator 1 is included. This simple phenomenon leads to the theory of central extensions of Lie algebras.

The next step in GAQ consists of finding a canonical association of group parameters with differential operators acting on complex functions defined on the group. This is accomplished with the help of the natural structure of rightinvariant vector fields, i.e., vector fields $X^{R}$ on the group invariant under the right multiplication $R_{a} g=g * a$. This is not the final step because the so-constructed operators usually provide only a reducible representation of the Lie algebra (Lie group) and therefore of the original Poisson algebra. Let us look at an example before going farther into the formalism.

The fundamental Poisson brackets for the three-dimensional free-particle are

$$
\begin{aligned}
& \left\{x^{i}, p_{j}\right\}=\delta_{j}^{i} 1, \quad\left\{x^{i}, H\right\}=p^{i} / m, \\
& \left\{p_{i}, H\right\}=0, \quad i j=1,2,3,
\end{aligned}
$$

and they must be mimicked by using a Lie group. The simplest group whose Lie algebra commutators are of the form (2.2) is a one-dimensional central extension $\widetilde{\mathbb{G}}_{m}$ of the rotationless Galilei group $\mathbb{G}$ parametrized by the mass $m$. The central subgroup may be either the multiplicative group $\mathrm{U}(1)$, parametrized by $\zeta \in \mathbb{C} /|\zeta|^{2}=1$, or the additive group $\mathbb{R}$, parametrized by the real number $\chi$. The group law is written as follows:

$$
\begin{aligned}
& B^{\prime \prime}=B^{\prime}+B, \quad B \in \mathbb{R}, \\
& \mathbf{A}^{\prime \prime}=\mathbf{A}^{\prime}+\mathbf{A}+\mathbf{V}^{\prime} B, \quad \mathbf{A} \in \mathbb{R}^{3}, \\
& \mathbf{V}^{\prime \prime}=\mathbf{V}^{\prime}+\mathbf{V}, \quad \mathbf{V} \in \mathbb{R}^{3}, \\
& \zeta^{\prime \prime}=\zeta^{\prime} \zeta \exp \left(i(m / \hbar)\left[\mathbf{A}^{\prime} \cdot \mathbf{V}+B\left(\mathbf{V}^{\prime} \cdot \mathbf{V}+\frac{1}{2} \mathbf{V}^{\prime 2}\right)\right]\right) .
\end{aligned}
$$

Since $B, \mathbf{A}$, and $\mathbf{V}$ are supposed to carry the dimensions of time, length, and velocity, respectively, a constant $\hbar$ with the dimensions of an action has to be introduced to render the exponent dimensionless. In (2.3) the group law for $\mathbb{G}$, $g^{\prime \prime}=g^{\prime} * g$, has been extended by the modified law $\xi^{\prime \prime}=\xi^{\prime} \xi \exp \left[i m \xi\left(g^{\prime}, g\right) / \hbar\right]$ of the (central) subgroup $U(1)$. The function $\xi\left(g^{\prime}, g\right)$ satisfies the so-called cocycle conditions:

$$
\begin{aligned}
& \xi\left(g^{\prime}, g\right)+\xi\left(g^{\prime} g, g^{\prime \prime}\right)-\xi\left(g^{\prime}, g g^{\prime \prime}\right)-\xi\left(g, g^{\prime \prime}\right)=0, \\
& \xi(e, e)=0,
\end{aligned}
$$

required to turn $\widetilde{\mathbb{G}}_{m}(g, \zeta) \equiv \widetilde{\mathbb{G}}_{m}(\tilde{g})$ into a true group law. The set of the $\xi$ 's that are not of coboundary form $\xi_{c}\left(g^{\prime} g\right)=\delta\left(g^{\prime} * g\right)-\delta\left(g^{\prime}\right)-\delta(g)$, for a real function $\delta$ on $\mathbb{G}$, constitutes an additive group $H^{2}(\mathbb{G}, \mathrm{U}(1))$ of dimension one, parametrized by the mass $m$. A coboundary can always be eliminated by a redefinition of $\zeta, \hat{\zeta}=\zeta e^{-i \delta}$.

Differentiating (2.3) from the left at the unity we get the right-invariant vector fields

$$
\begin{aligned}
& \widetilde{X}_{(B)}^{R}=\frac{\partial}{\partial B}, \\
& \widetilde{X}_{\left(A^{i}\right)}^{R}=\frac{\partial}{\partial A^{i}}+\frac{m}{\hbar} V_{i} \Xi, \\
& \widetilde{X}_{\left(V^{\prime}\right)}^{R}=\frac{\partial}{\partial V^{i}}+B \frac{\partial}{\partial A^{i}}+\frac{m}{\hbar} B V_{i} \Xi, \\
& \widetilde{X}_{(\zeta)}^{R}=i \zeta \frac{\partial}{\partial \zeta} \equiv \Xi,
\end{aligned}
$$


from which the operators associated with the quantities $B$, $\mathbf{A}, \mathbf{V}$, and $\zeta$ must be derived. Their commutation relations are

$$
\begin{aligned}
& {\left[\widetilde{X}_{(B)}^{R}, \widetilde{X}_{\left(A^{\prime}\right)}^{R}\right]=0, \quad\left[\widetilde{X}_{(B)}^{R}, \widetilde{X}_{\left(V^{(}\right)}^{R}\right]=\widetilde{X}_{\left(A^{\prime}\right)}^{R},} \\
& {\left[\widetilde{X}_{\left(A^{\prime}\right)}^{R}, \widetilde{X}_{\left(A^{\prime}\right)}^{R}\right]=0, \quad\left[\widetilde{X}_{\left(V^{\prime}\right)}^{R}, \widetilde{X}_{\left(V^{\prime}\right)}^{R}\right]=0,} \\
& {\left[\widetilde{X}_{\left(V^{\prime}\right)}^{R}, \widetilde{X}_{\left(A^{\prime}\right)}^{R}\right]=(m / \hbar) \delta_{i j} \Xi .}
\end{aligned}
$$

The fields in (2.5) act as derivatives on complex functions $\psi$ on $\widetilde{\mathbb{G}}_{m}$ satisfying the U(1)-equivariance condition:

$$
\Xi . \psi=i \psi \Leftrightarrow \psi(\zeta * \tilde{g})=\zeta * \psi(\tilde{g}),
$$

which factors out the dependence on the added variable $\zeta$.

This completes the Bohr approximation to quantization. The procedure has to be continued because the representation of (2.2) by (2.5) acting on the $\psi$ 's of (2.7) is reducible. A proof of that is the existence of nontrivial operators commuting with the representation, i.e., with each one of the operators in (2.5). The vector fields

$$
\frac{\partial}{\partial \mathbf{A}}, \frac{\partial}{\partial \boldsymbol{B}}+\mathbf{V} \cdot \frac{\partial}{\partial \mathbf{A}}+\frac{1}{2 \hbar} m \mathbf{V}^{2} \Xi
$$

are examples of such operators and reducing the representation requires to kill them on $\psi$. The true wave functions are complex functions on $\widetilde{\mathbb{G}}_{m}$ such that

$$
\begin{aligned}
& \Xi \cdot \psi=i \psi, \\
& \frac{\partial \psi}{\partial \mathbf{A}}=0, \\
& \frac{\partial \psi}{\partial B}+\mathbf{V} \cdot \frac{\partial \psi}{\partial \mathbf{A}}+\frac{i}{2 \hbar} m \mathbf{V}^{2} \psi=0,
\end{aligned}
$$

which means $\psi=\xi \Phi(B, \mathbf{V})$, with $\Phi$ such that $i \hbar \partial \Phi / \partial B=\left(m V^{2} / 2\right) \Phi$, i.e., the Schrödinger equation in momentum space.

The vector fields (2.8) turn out to be a subalgebra of the algebra of left-invariant vector fields $\mathscr{X}^{L}\left(\widetilde{\mathbb{G}}_{m}\right)$, on $\widetilde{\mathbb{G}}_{m}$ :

$$
\begin{aligned}
& \widetilde{X}_{(B)}^{L}=\frac{\partial}{\partial B}+\mathbf{V} \cdot \frac{\partial}{\partial \mathbf{A}}+\frac{m \mathbf{V}^{2}}{2 \hbar} \Xi, \\
& \widetilde{X}_{(\mathbf{A})}^{L}=\frac{\partial}{\partial \mathbf{A}}, \quad \mathbf{X}_{(\mathbf{V})}^{L}=\frac{\partial}{\partial \mathbf{V}}+\frac{1}{\hbar} m \mathbf{A} \Xi \\
& \widetilde{X}_{(\zeta)}^{L}=i \xi \frac{\partial}{\partial \xi} \equiv \Xi .
\end{aligned}
$$

The vector fields (2.8) constitute the maximal left subalgebra containing half the generators appearing in the left ana$\log$ of the commutator (2.6b) and the kernel of the Lie algebra cocycle, i.e., the linear mapping $\Sigma: \mathscr{G} \times \mathscr{G} \rightarrow \mathbb{R}$ assigning $m / \hbar$ to the pair $\left(X_{\left(V^{i}\right)}^{R}, X_{\left(A^{i}\right)}^{R}\right)$ and 0 otherwise. This sort of subalgebra will be called in the sequel full polarization subalgebra $\mathscr{P}$ and generalizes the concept of polarization in standard geometric quantization ${ }^{13}$ in that it contains the kernel of $\Sigma$ to be referred to as the characteristic subalgebra.

The Lie algebra cocycle $\Sigma$ is the algebraic generalization of the symplectic form of the Cartan formalism. ${ }^{20}$ In fact, $\Sigma$ can be substituted by a differential two-form on $\widetilde{\mathbb{G}}_{m}$ taking the value $\Sigma$ at the unity. To do that we take the left-invariant canonical one-form $\vartheta^{L}$ on $\widetilde{\mathbb{G}}_{m}$,

$\vartheta=\vartheta^{L(B)} \widetilde{X}_{(B)}^{L}+\vartheta^{L(A)} \widetilde{X}_{(A)}^{L}+\vartheta^{L(\mathrm{~V})} \widetilde{X}_{(\mathrm{V})}^{L}+\vartheta^{L(\zeta)} \widetilde{X}_{(\zeta)}^{L}$.
It satisfies $\vartheta^{L}\left(\widetilde{X}^{L}\right)=\widetilde{X}^{L}, \forall \widetilde{X}^{L} \in \mathscr{X}^{L}\left(\widetilde{\mathbb{G}}_{m}\right)$. Then we select the $\mathrm{U}(1)$ component (vertical component) $\vartheta^{L(\zeta)} \equiv \Theta$, the quantization form, and $\left.d \Theta\right|_{e}=\Sigma$. A trivial calculation shows that

$$
\theta=-m \mathbf{A} \cdot d \mathbf{V}-\left(m \mathbf{V}^{2} / 2\right) d B+\hbar d \zeta / i \zeta
$$

The one-form $\theta$, which is invariant under the right-invariant vector fields $(2.5), \quad d \Theta\left(\widetilde{X}^{R}\right)+d\left(\Theta\left(\widetilde{X}^{R}\right)\right)$ $\equiv\left(i_{\hat{X}^{R}} d+d i_{\hat{X}^{R}}\right) \Theta \equiv L_{\tilde{X}^{R}} \Theta=0$, generalizes the CartanHilbert-Poincaré one-form of the variational calculus and defines the Noether invariants

$$
\begin{aligned}
& -i_{\tilde{X}_{i s}^{R}} \Theta=m \mathbf{V}^{2} / 2, \quad i_{\tilde{X}_{(A)}^{R}} \Theta=m \mathbf{V}, \\
& -i_{\tilde{X}_{i, r}^{R}} \Theta=m \mathbf{A}-m \mathbf{V} B .
\end{aligned}
$$

Our generalized Poisson brackets

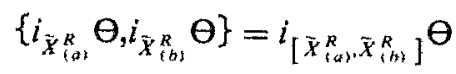

reproduce the initial ones $(2.2)$.

The classical "limit" appears as the dynamical group $\overline{\mathrm{G}}_{m}$ corresponding to a central extension of $\mathbb{G}$ by the additive group $\mathbb{R}$. The replacement consists in substituting a real function $S$ for $\Psi$,

$$
\chi^{\prime \prime}=\chi^{\prime}+\chi+m\left[\mathbf{A} \cdot \mathbf{V}+B\left(\mathbf{V}^{\prime} \cdot \mathbf{V}+\frac{1}{2} \mathbf{V}^{\prime 2}\right)\right],
$$

for the last line in (2.3) and

$$
\Xi: S=1 \Leftrightarrow S(\chi+\bar{g})=\chi+S(\bar{g}),
$$

for (2.7). Now the $\hbar$ constant is no longer needed as $\chi$ may carry the dimensions of an action. The effect of $(2.16)$ can be seen when writing down the "classical operator" $\bar{X}^{R}$ acting on the "wave function" $S$. We get for instance $\overline{\mathrm{X}}_{(\mathrm{A})}^{R} \cdot S=m \mathrm{~V}$ instead of $-i \hbar \widetilde{X}_{(\mathrm{A})}^{R} \cdot \psi=m \mathrm{~V} \psi$ and similar expressions for other generators. For the polarization conditions a difference also appears:

$$
\bar{X}_{(B)}^{L} \cdot S=0 \Rightarrow \frac{\partial S}{\partial B}+\frac{m V^{2}}{2}=0,
$$

which is nothing other than the Hamilton-Jacobi equation in momentum space. More details about the free-particle as well as other examples can be found in Refs. 21 and 3.

The procedure so far followed can be repeated with general groups or starting with a general set of (perhaps deformed) Poisson brackets. In Ref. 22 general formulas are given to reconstruct a group law from a set of structure constants. Here, in Sec. IV we will have the opportunity of dealing with a nontrivial set of (super) Poisson brackets.

Let us go briefly to the gencral situation of a Lie group $\widetilde{G}$ before discussing the constrained case which presents an additional generalization of GAQ.

A quantum group is a Lie group $\widetilde{G}$ that in turn is a right principal bundle with structure group $U(1)$ or $\mathbb{R}$, with a oneform $\Theta$ naturally selected among the left-invariant oneforms. ${ }^{2,3,22}$

The structure of right principal bundle ${ }^{23}$ of $\widetilde{G}$ means that its group law can be written in such a way that no unprimed $U(1)$ (or $\mathbb{R}$ ) parameter appears in the group law equations except in the one for $U(1)$ (or $\mathbb{R}$ ). That insures that $U(1)$ (or $\mathbb{R}$ ) only acts from the right on the remainder (remember that we write for the group law $g^{\prime \prime}=g^{\prime} * g$ ). The 
case of a central extension is only a particular situation corresponding to a physical system with classical limit.

The one-form $\Theta$, dual to the fundamental vector field $\widetilde{X}_{(\zeta)}^{L} \in \mathscr{X}^{L}(\widetilde{G}) \approx \widetilde{G}$ [for the sake of brevity we will think of a structure group $U(1)$ parametrized by $\zeta ; \mathscr{X}^{L}(\widetilde{G})$ is the Lie algebra of left-invariant vector fields and $\widetilde{G} \equiv \mathscr{Q}^{L}(e)$ is the usual Lie algebra], is the quantization form and plays the role of a left-invariant connection form on the principal bundle $\widetilde{G} \rightarrow \widetilde{G} / \mathrm{U}(1)$. The curvature $\Omega=\mathrm{d} \Theta$ is a presymplectic form (generalizing the symplectic form of the Cartan calcu$\operatorname{lus}^{20}$ ). Unlike a proper symplectic form $\mathrm{d} \Theta$ has a kernel. We call $\mathscr{C}_{\Theta} \equiv$ kernel $\Theta \cap$ kernel $d \Theta$ characteristic module of $\Theta$ and it turns out to be generated by a basis of a subalgebra (the characteristic subalgebra) $\mathscr{G}_{\theta}$ of $\mathscr{Q}^{L}(\widetilde{G})$. The quotient $(\widetilde{G}, \Theta) / \mathscr{C}_{\Theta}$ is a quantum manifold in the standard geometric quantization sense ${ }^{13}$ although we need not take any quotient at all. Indeed the trajectories of the vector fields in $\mathscr{G}_{\theta}$ are the generalized equations of motion. ${ }^{2,21}$ The Noether invariants are given by the right-invariant vector fields $\mathscr{Z}^{R}(\widetilde{G})$ through the expression $i_{\widetilde{X}_{(a)}^{R}} \Theta \equiv \mathfrak{H}$.

A full polarization (polarization) $\mathscr{P}$ is a maximal horizontal left subalgebra of $\widetilde{G}$ containing $\mathscr{G}_{\Theta}$ (a subalgebra of $\left.\mathscr{Y}_{\theta}\right)$. The horizontality is naturally defined in terms of the connection form $\theta$ and means that $\Theta$ is zero on any vector field in $\mathscr{P}$. This prevents $\mathscr{P}$ from containing any co-ordinate-momentum pair. The nonexistence of full polarizations is related to the presence of an anomaly. ${ }^{24}$

Wave functions are (complex) functions on $\widetilde{G}$ that satisfy the $U(1)$-equivariance condition from the left (2.7) and the full polarization condition

$$
\widetilde{X}^{L} \cdot \psi=0 \quad \forall \widetilde{X}^{L} \in \mathscr{P} .
$$

Finally, the physical operators are the right-invariant vector fields $\widetilde{X}^{R} \in \mathscr{X}^{R}(\widetilde{G})$ acting on $\psi$ as derivations. We will not be concerned here with scalar products nor similar details that are found in Ref. 21.

Note that the group representations obtained in this way are not necessarily irreducible, although reducing them is now very simple because we know the explicit form of the wave functions. For instance, in the infinite-dimensional case (also for noncompact finite-dimensional groups) one finds representations that are reducible but not a direct product of irreducible components. The full reduction requires defining a vacuum from which the right vectors (operators) generate automatically the (irreducible) Fock space $\mathscr{H}$ (Ref. 4). A vacuum is a polarized wave function that is annihilated by a right polarization (e.g., the right version of a polarization $\left.: \mathscr{P}^{\prime} \equiv \mathscr{P}^{\prime L}\right)$. When this right polarization is the right version $\mathscr{P}^{R}$ of the polarization defining the actual quantization, the vacuum turns out to be a Dirac-like vacu$u m$. We refer to Ref. 4 where it is shown that the space of polarized functions on the Virasoro group, although it does not contain any null vector, is bigger than the irreducible Fock space.

The generalization of the present formalism allowing for constraints ${ }^{3}$ is quite simple and consists in enlarging the structure subgroup of $\widetilde{G}$. Taking a subgroup $T$ bigger than $U(1)$ (which for physically interesting cases will contain the
$\mathrm{U}$ (1) group of phase invariance) requires a generalization of (2.7) taking the general form,

$$
\Psi\left(\tilde{g}_{T} * \tilde{g}\right)=D\left(\tilde{g}_{T}\right) \psi \Leftrightarrow \widetilde{X}^{R} \psi=d D(\widetilde{X}) \psi, \quad \widetilde{X} \in \mathscr{T},
$$

where $D(d D)$ is a particular representation of $T(\mathscr{T})$ characterizing the quantization. Of course $\psi$ takes its values on the representation space of $T$. One condition in (2.19) corresponds to the $U(1)$ equivariance, and the others are related to constraints in the usual sense. In order to allow the theory for general representations $D$, in particular one-dimensional ones, (2.19) must be restricted to a particular subalgebra of $\mathscr{T}$. As a subgroup of $\widetilde{G}, T$ may contain some co-ordinatemomentum pair in addition to the $T_{0}[=\mathrm{U}(1)]$ subgroup and a full polarization subgroup $T_{P}$ can be defined much in the same way we defined $\mathscr{P}$ (or its corresponding group) inside $\widetilde{G}$. The actual generalization of $(2.7)$ then consists in replacing the group $T$ in (2.19) by the minimal subgroup containing $T_{0}$ and $T_{P}$. Once a polarization $\mathscr{P}$ of $\widetilde{G}$ has been fixed we must select the subgroup $T_{P}$ in such a way that its algebra be contained in the (left) polarization $\mathscr{P}^{\prime}$ whose right version define the vacuum. Otherwise a trivial result is, in general, obtained. More precisely, the algebra $\mathscr{T}$ in (2.19) must be restricted to the subalgebra of $\mathscr{T}$, generated by

$$
\left\langle\mathscr{P}^{\prime} \cap \mathscr{T}, \mathscr{T}_{0}\right\rangle,
$$

where $\mathscr{T}_{0}$ is the generator of $T_{0}$.

To compare at this point with the Dirac theory of constraints we have to restrict ourselves to the case of a group with the classical limit or, more precisely, to a central extension. The unconstrained phase space can be recovered as the manifold $M=\widetilde{G} /\left\langle\mathscr{G}_{\theta}\right\rangle / T_{0}$. The constraint surface of $M$ would be defined by the equations $i_{\widetilde{X}^{R}} \Theta=0, \widetilde{X}^{R} \in \mathscr{T} / T_{0}$. That submanifold could be in general nonsymplectic (corresponding to first class constraints) and a further quotient by the kernel of the presympletic form is called for.

It must be noted that the constraint (2.19), which is nothing other than a $T$-equivariance condition, is written in terms of right-invariant vector fields (remember that they generated finite left translations) although the bundle $\widetilde{G}$ with structure group $T$ is a right principal bundle. Unlike the polarization condition it does not commute, in general, with the physical operators. Therefore, only a subalgebra of $\mathscr{Z}^{R}(\widetilde{G})$, that of good operators, stabilizes the space of wave functions (see Ref. 3 for details).

\section{A SIMPLE EXAMPLE: NON-ABELIAN CONSTRAINTS ON THE FREE-PARTICLE}

We start again with the Galilei group but this time including rotations [parametrized by $\epsilon$ such that $|\boldsymbol{\epsilon}|=2 \sin (\varphi / 2)]$. The formula (2.3) is replaced by

$t^{\prime \prime}=t^{\prime}+t, \quad t \in \mathbb{R}$,

$\mathbf{x}^{\prime \prime}=\mathbf{x}^{\prime}+R^{\prime}(\epsilon) \mathbf{x}+\mathbf{v}^{\prime} t, \quad \mathbf{x} \in \mathbb{R}^{3}$,

$\mathbf{v}^{\prime \prime}=\mathbf{v}^{\prime}+R^{\prime}(\epsilon) \mathbf{v}, \quad \mathbf{v} \in \mathbb{R}^{3}$,

$\epsilon^{\prime \prime}=\sqrt{\left(1-\epsilon^{\prime 2} / 4\right)} \epsilon+\sqrt{\left(1-\epsilon^{2} / 4\right)} \epsilon^{\prime}-\epsilon^{\prime} \times \epsilon / 2, \quad \epsilon \in \mathbb{R}^{3}$, 


$$
\begin{aligned}
& \zeta^{\prime \prime}=\zeta^{\prime} \zeta \exp \left(i m\left[\mathbf{x}^{\prime} R^{\prime} \mathrm{v}+t\left(\mathrm{v}^{\prime} R^{\prime} \mathrm{v}+\mathrm{v}^{\prime 2} / 2\right)\right] / \hbar\right), \\
& \zeta \in \mathrm{U}(1), \\
& R(\epsilon)_{j}^{i}=\left(1-\epsilon^{2} / 2\right) \delta_{j}^{i}+\sqrt{\left(1-\epsilon^{2} / 4\right)} \eta_{\cdot j k}^{i} \epsilon^{k}+\frac{1}{2} \epsilon^{i} \epsilon_{j},
\end{aligned}
$$

where a more physical notation has now been chosen. The application of the GAQ to $\widetilde{\mathbb{G}}_{m} \rightarrow \widetilde{\mathbb{G}}_{m} / \mathrm{U}(1)[U(1)$ is the structure group], as in Sec. II, leads to the same results as before with the only modification that $\mathscr{G}_{\theta}$ (and therefore $\mathscr{P}$ ) contains the whole subgroup SU(2). The added polarization condition equations $\widetilde{X}_{(\epsilon)}^{L} \cdot \psi=0$ just say $\psi \neq \psi(\epsilon)$. In fact, the left-invariant vector fields associated with $\epsilon$ are

$$
\widetilde{X}_{\left(\epsilon^{\prime}\right)}^{L}=\left[\sqrt{\frac{1-\epsilon^{2}}{4}} \delta_{j}^{i}+\frac{\eta_{\cdot j k}^{i} \epsilon^{k}}{2}\right] \frac{\partial}{\partial \epsilon^{i}}
$$

and the matrix $\widetilde{X}_{\left(\epsilon^{\prime}\right)}^{L \epsilon^{\prime}}$ has an inverse, so that $\widetilde{X}_{\left(\epsilon^{\prime}\right)}^{L \epsilon^{\prime}}\left(\partial \psi / \partial \epsilon^{j}\right)=0$ implies $\partial \Psi / \partial \epsilon^{i}=0$.

The right-invariant vector ficlds $\widetilde{X}_{\left(\epsilon^{\prime}\right)}^{R}$,

$$
\begin{aligned}
\widetilde{X}_{\left(\epsilon^{j}\right)}^{R}= & {\left[\sqrt{\frac{1-\epsilon^{2}}{4}} \delta_{j}^{i}-\frac{\eta_{\cdot j k}^{i} \epsilon^{k}}{2}\right] \frac{\partial}{\partial \epsilon^{i}} } \\
& -\eta_{\cdot j k}^{i} x^{k} \frac{\partial}{\partial x^{i}}-\eta_{\cdot j k}^{i} v^{k} \frac{\partial}{\partial v^{k},}
\end{aligned}
$$

have, however, a nontrivial action on $\psi$, since although $\psi \neq \neq(\boldsymbol{\epsilon}, \mathbf{x})$ a term in $\partial / \partial \mathbf{v}$ still remains which gives the orbital angular momentum operator.

Let us now consider the right principal bundle structure (see the comment in the previous section) on $\widetilde{\mathbb{G}}_{m}, \widetilde{\mathbb{G}}_{m}$ $\rightarrow \widetilde{\mathbb{G}}_{m} /[\mathrm{SU}(2) \otimes \mathrm{U}(1)]$. This means that the equivariance conditions are now different. They are

$$
\begin{aligned}
& \widetilde{X}_{(5)}^{R} \cdot \psi \equiv \Xi \cdot \Psi=i \psi, \\
& \widetilde{X}_{(\mathrm{E})}^{R} \cdot \psi=d D(\mathbf{J}) \psi,
\end{aligned}
$$

where $d D\left(J^{i}\right)$ is the representative of the abstract rotation generator along $\epsilon^{i}$.

As in the unconstrained case the characteristic subalgebra of the quantization form $\vartheta^{L(\xi)}$ is generated by $\left(\bar{X}_{(t)}^{L}, \widetilde{X}_{(\epsilon)}^{L}\right)$ and the polarization one by $\left(\widetilde{X}_{(t)}^{L}, \widetilde{X}_{(x)}^{L}, \widetilde{X}_{(\epsilon)}^{L}\right)$. Thus the full polarization condition (2.8) plus (3.4a) say

$\left(\tilde{X}_{(\zeta)}^{R} \Rightarrow\right), \quad \psi(\zeta, \mathbf{x}, t, \mathbf{p}, \boldsymbol{\epsilon})=\zeta \psi(\mathbf{x}, t, \mathbf{p}, \mathbf{\epsilon}), \mathbf{p}=m \mathbf{v}$,

$\left(\widetilde{X}_{(\mathbf{e})}^{L}, \widetilde{X}_{(\mathbf{x})}^{L} \Rightarrow\right), \quad \psi(\mathbf{x}, t, \mathbf{p}, \mathbf{\epsilon})=\psi(t, \mathbf{p})$,

$\left(\widetilde{X}_{(t)}^{L} \Rightarrow\right), \quad \psi(t, \mathbf{p})=\exp \left\{-i \mathbf{p}^{2} t / 2 m \hbar\right\} \varphi(\mathbf{p}), \quad \forall \varphi(\mathbf{p})$,

where the condition $(3.4 \mathrm{~b})$ is still left.

Radial motion: Take for $d D$ in (3.4b) the trivial representation $d D(\mathbf{J})=0$. The condition (3.4b) on the arbitrary function $\varphi(\mathbf{p})$ tell us

$$
\mathbf{p} \times \frac{\partial \varphi}{\partial \mathbf{p}}=0 \Rightarrow \varphi(\mathbf{p})=\varphi(|\mathbf{p}|) .
$$

"l-wave" motion: Now $d D$ will be the irreducible representation of $\mathrm{SU}(2)$ with integer index $l, \mathrm{dD}^{(l)}$. The wave function is made out of $2 l+1$ identical copies of the until now arbitrary function $\varphi(\mathbf{p}), \varphi^{(l)}: \widetilde{\mathbb{G}}_{m} \rightarrow \mathbb{C}^{2 l+1}$. Equation (3.4b) implies

$$
\mathbf{p} \times \frac{\partial \varphi_{m}^{(l)}}{\partial \mathbf{p}}=\sum_{m^{\prime}} d D_{m m^{\prime}}^{(l)}(\mathbf{J}) \varphi_{m^{\prime}}^{(l)}
$$

i.e., $\varphi_{m}^{(l)}(\mathrm{p})$ are the $2 l+1$ components of the momentum wave function of the free-particle moving in $l$-wave.

\section{IV. "BRST" SUPERGROUP}

The BRST transformation ${ }^{5}$ was introduced in gauge field theory as a powerful tool to derive the Ward identities. ${ }^{26}$ It has the form of a generalized gauge transformation, (generated by a fermionic parameter) which leaves invariant the effective Lagrangian including the gauge fixing and the Faddeev-Popov ghost terms. ${ }^{26}$

Using the BRST transformation as an essential ingredient, Batalin, Fradkin, and Vilkovisky ${ }^{6}$ have developed in a series of papers a generalized quantization method for arbitrary Hamiltonian constrained systems. In a path integral approach they construct a generalized BRST-invariant Hamiltonian (containing new bosonic and fermionic variables) such that the resulting $S$-matrix is gauge invariant and unitary on the subspace of physical states. The important feature of this method is that it does not require the closure of the gauge algebra off-shell. The method has also been applied to (super)gravity and (super)string theory, where the Virasoro group plays (in a sense) the role of an infinite-dimensional gauge group.

In a geometrical analysis of the Yang-Mills case, the ghost fields were interpreted as Maurer-Cartan forms on the infinite-dimensional group $B$ of gauge transformations and the BRST operator as exterior differential forms on $\$$ (Ref. 27).

A complete cohomological interpretation of the classical BRST formalism for the case of a true gauge theory is due to McMullan. ${ }^{7}$ There, antighosts arise as part of the Koszul complex that encodes the fact that the dynamical system is restricted (by the constraint equations) to a submanifold of the phase space. Ghosts arise in the gauge group cohomology to describe the fact that points along gauge orbits on the constrained submanifold are physically equivalent. The BRST operator is the differential operator of the resulting double complex.

Henneaux ${ }^{28}$ has elaborated on the classical aspects of Hamiltonian BRST theory and shown that the existence of a BRST operator is related to the existence of a series of higher-order structure functions (associated with a given set of constraint functions) satisfying certain identities of Jacobi type.

Using homological algebra tools, the existence of a BRST operator can be shown for a large class of constrained systems, ${ }^{29}$ in particular, for reducible gauge theories, where the constraints are no longer independent and hence more than one generation of ghosts is needed..$^{30}$

For an operator based, but not necessarily standard quantization of these classical structures one would like to translate the BRST cohomological description into differential geometric language. A geometric model for the extended phase space of the BRST approach for the Lie algebra case has been given in Ref. 8 in terms of a fiber bundle over the phase space $S$ with fiber $\mathscr{L}(G) \oplus \mathscr{L}^{*}(G)$, the direct sum of the gauge Lie algebra and its dual. The structure group of this supermanifold is the orthosymplectic group which the BRST operator "breaks" down to the symplectic structure 
group of the physical reduced phase space. A complete geometrical formulation is needed to get some control over what is happening off the constraint surface - a fact that is expected to be of importance in any quantum theory-and to throw some light on the question of whether the case of an open "gauge algebra" is fundamentally different from the case of a true gauge algebra of constraints.

Our approach here is rather different in that we will not attempt to explain the "naturality" of the fermionic variables present in the formalism. On the contrary, our starting point is an associative formal group law ${ }^{31}$ (a straightforward generalization of a local group law) whose variables may easily be turned into fermionic ones. As far as this mathematical structure is concerned, fermionic parameters require no special motivation and our scheme is simpler in structure than those involving differential forms on a bialgebra joined to the unconstrained solution manifold $S$, etc. Moreover, and this is perhaps the most important feature from the physical point of view, the treatment of general systems will be quite similar to that of the free-particle or the harmonic oscillator. In all these cases we look for a supergroup from which the adequate (quantum) constrained dynamics is obtained with the help of the GAQ formalism.

First of all we will concentrate on that part of the supergroup which is closely related to the constraint subgroup $K$, disregarding the ordinary variables of $t, x$, and $p$ type. Hence, our results will apply to any physical system whose constraint subgroup is known and we will express all formulas in terms of the group law for the bosonic subgroup $K$ and the adjoint (and coadjoint) representation of its Lie algebra $\mathscr{K}$.

The smallest supergroup $\widetilde{B}(K)$ containing $K$, the transformations generated by the BRST charge and the ghost and antighost operators contains additional bosonic transformations. ${ }^{1}$ In fact, although the operators $\hat{k}_{i}, \hat{Q}, \widehat{\Psi}_{j}$ generate an algebra, ${ }^{32}$ the one generated by $\hat{k}_{i}, \hat{Q}, \hat{\Psi}_{j}, \stackrel{\Psi}{* k}^{* k}, i, j, k=1, \ldots$, $\operatorname{dim} K$ does not close as can be seen by using the standard formulas:

$$
\begin{aligned}
& {\left[\hat{k}_{i}, \hat{k}_{j}\right]=f_{i j}{ }^{k} \hat{k}_{k}, \quad\left[\hat{k}_{i}, \hat{\Psi}_{j}\right]=f_{i j}{ }^{m} \hat{\Psi}_{m},} \\
& \left\{\hat{\Psi}_{1}, \hat{\Psi}^{j *}\right\}=\lambda \delta_{i}^{j} \hat{\mathbf{1}}, \quad\left[\hat{k}_{i}, \hat{\Psi}^{* j}\right]=-f_{i m} j \hat{\Psi}^{* m}, \\
& \left\{\hat{Q}, \hat{\Psi}_{i}\right\}=\hat{k}_{i},
\end{aligned}
$$

where

$$
\widehat{Q}=\hat{\Psi}^{* i} \hat{k}_{i}+\frac{1}{2} f_{m n} \hat{\Psi}_{i} \hat{\Psi}^{* m} \hat{\Psi}^{* n}, \quad \lambda \in \mathbb{R}
$$

[see the comment after (4.10) concerning the relative + sign].

The Jacobi identities reveal the need for a new set of bosonic operators $\hat{T}^{i}$ to account for the anticommutators $\left\{\hat{Q}, \hat{\Psi}^{* \prime}\right\}$. These new operators were already considered in Ref. 33 as additional conceivable BRST invariant operators. They will play an important role in our theory (see later on in this section). Adding the new generators

$$
\widehat{T}^{i} \equiv\left\{\widehat{Q}, \hat{\Psi}^{* i}\right\},
$$

(4.1a) is completed with the following commutators:

$$
\begin{aligned}
& {\left[\hat{Q}, \hat{T}^{i}\right]=0, \quad\left[\widehat{T}^{i}, \widehat{T}^{j}\right]=0, \quad\left[\widehat{T}^{i}, \hat{\Psi}^{* j}\right]=0,} \\
& {\left[\hat{\Psi}_{j}, T_{i}\right]=-f_{j m} \hat{\Psi}^{* m}, \quad\left[\hat{k}_{i}, \widehat{T}^{j}\right]=-f_{i m} \widehat{T}^{m} .}
\end{aligned}
$$

A remarkable property of $\widetilde{\mathfrak{B}}(K)$ is that no anomalous term (nontrivial central extension) is allowed for the subalgebra $\mathscr{K}$ as a consequence of the anticommutator $\left\{Q, \hat{\Psi}_{i}\right\}=\hat{k}_{i}$. In fact, $\widetilde{\mathfrak{B}}(K)$ is the simplest quantum supergroup which forbids any possible central extension of the constraint subgroup. The fermionic character of $\hat{Q}, \hat{\Psi}$, and $\hat{\Psi}^{*}$ is a consequence of this requirement. In spite of these considerations the subgroup generated by $\hat{K}, \hat{\Psi}, \hat{\Psi}^{*}$ could admit a (negative) anomaly, for instance when $K$ is diff $S^{1}$. Another interesting property concerns the new generators $\widehat{T}_{i}$. Although they do not have any dynamical content (in the sense that no central term appears on the rhs of $\left.\left[\widehat{T}_{i}, \widehat{T}_{j}\right]\right)$, the subgroup of these new generalors affects the definition of the physical vacuum. Of course $\widetilde{\mathfrak{B}}(K)$ can be enlarged so as to contain the actual set of coordinate-momentum pairs of the physical system under consideration (see Sec. VI).

Our task now consists in finding a supergroup law on the set of variables $k^{i}, q, \Psi^{j}, \Psi_{k}^{*}, T_{m}, \zeta$ in such a way that the relations (4.1) correspond to the commutators of the, say, right-invariant vector fields $\widetilde{X}_{\left(k^{\prime}\right)}^{R}, \widetilde{X}_{(q)}^{R}, \widetilde{X}_{\left(\psi^{\prime}\right)}^{R}, \widetilde{X}_{\left(\Psi_{k}^{*}\right)}^{R}$, $\widetilde{X}_{\left(T_{m}\right)}^{R}$, and $\widetilde{X}_{(\zeta)}^{R}$. Note that $\widetilde{\mathcal{B}}(K)$ has the structure of a quantum supergroup, indeed, it is a central extension parametrized by $\lambda$, with dynamical variables $\Psi^{i}, \Psi_{j}^{*}$.

We now give the supergroup law of $\widetilde{\mathfrak{B}}(K)$ for a general constraint group $K$ with vector realization $R_{{ }_{j}}^{i}$ :

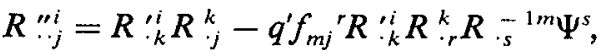

$$
\begin{aligned}
& \Psi^{\prime \prime j}=\Psi^{\prime j}+R_{\cdot k}^{\prime j} \Psi^{k}-\frac{1}{2} q^{\prime} f_{k s}^{j} R_{{ }_{m}}^{\prime k} R^{\prime s}{ }_{n} \Psi^{m} \Psi^{n} \text {, } \\
& \Psi_{j}^{* \prime \prime}=\Psi_{j}^{* \prime}+R_{\cdot j}^{\prime-1 k} \Psi_{k}^{*}-f_{j s}{ }^{k} R_{k}^{{ }^{-1 m}} \Psi^{\prime s} T_{m} \\
& +q^{\prime} f_{j s}{ }^{n} R^{\prime}{ }_{n}^{-1 k} \Psi^{\prime s} \Psi_{k}^{*}, \\
& T_{j}^{\prime \prime}=T_{j}^{\prime}+R_{\cdot j}^{\prime-1 k} T_{k}+q^{\prime} R_{j}^{\prime-1 k} \Psi_{k}^{*}, \quad q^{\prime \prime}=q^{\prime}+q, \\
& \zeta^{\prime \prime}=\zeta^{\prime} \zeta \exp i(\lambda / 2)\left\{\Psi_{i}^{* \prime} R^{\prime i} \cdot{ }_{j} \Psi^{j}-\Psi_{i}^{*} R_{\cdot j}^{\cdot-1 i} \Psi^{\prime j}\right. \\
& +f_{j k}{ }^{i}:_{i}^{i^{-1 m}} T_{m} \Psi^{\prime j} R:_{s}^{k} \Psi^{s}+\frac{1}{2} q^{\prime} f_{j k}{ }^{i} \Psi^{j} \Psi^{k} \Psi_{i}^{*} \\
& +\frac{1}{2} q^{\prime} f_{j k}^{i} R_{\cdot i}^{\prime m} \Psi_{m}^{* \prime} \Psi^{j} \Psi^{k}+q^{\prime} f_{j k}^{i} \Psi^{\prime j} R_{m_{m}^{\prime}}^{\prime k} \Psi^{m} R_{i_{i}}^{\prime-1} \Psi_{s}^{*} \\
& \left.+\frac{1}{2} q^{\prime} f_{j k}{ }^{i} f_{m n}{ }^{r} !_{\cdot i}^{-1}{ }^{-1 s} R^{\prime}{ }_{\cdot r} \Psi^{\prime j} \Psi^{m} \Psi^{n} T_{s}\right\}
\end{aligned}
$$

From (4.3) the left- and right-invariant vector fields

$$
\widetilde{X}_{(a)}^{L}=\left.\frac{\partial \tilde{g}^{\prime \prime b}}{\partial \tilde{g}^{a}}\right|_{\tilde{g}=e} \frac{\partial}{\partial \tilde{g}^{\prime b}}, \quad \widetilde{X}_{(a)}^{R}=\left.\frac{\partial \tilde{g}^{\prime \prime b}}{\partial \tilde{g}^{\prime a}}\right|_{\tilde{g}^{\prime}=e} \frac{\partial}{\partial \tilde{g}^{b}}
$$

are easily derived keeping an eye on the even or odd character of the variables. They are

$$
\begin{aligned}
& \widetilde{X}_{(q)}^{L}=\frac{\partial}{\partial q}, \quad \widetilde{X}_{\left(k^{j}\right)}^{L}=X_{\left(k^{\prime}\right)}^{L_{k} j} \frac{\partial}{\partial k^{j}}, \\
& \widetilde{X}_{\left(\Psi^{\prime}\right)}^{L}=R^{j}{ }_{i} \frac{\partial}{\partial \Psi^{j}}-q X_{\left(k^{\prime}\right)}^{L_{k} j} \frac{\partial}{\partial k^{j}}-\frac{\lambda}{2} R_{. i}^{j} \Psi^{*}{ }_{j} \Xi, \\
& \widetilde{X}_{\left(\Psi_{i}^{*}\right)}^{L}=R_{. j}^{-1 i} \frac{\partial}{\partial \Psi_{j}^{*}}-q R_{. j}^{-1 i} \frac{\partial}{\partial T_{j}}-q R_{. n}^{-1 i} f_{s j}^{n} \\
& \times \Psi^{s} \frac{\partial}{\partial \Psi_{j}}-\frac{\lambda}{2} R_{. j}^{-1 i} \Psi^{j} \Xi
\end{aligned}
$$




$$
\begin{aligned}
& \widetilde{X}_{\left(T_{i}\right)}^{L}=R_{, j}^{-1 i} \frac{\partial}{\partial T_{j}}+R_{. j}^{-1 i} f_{m n}^{j} \Psi^{n} \frac{\partial}{\partial \Psi_{m}^{*}}, \\
& \tilde{X}_{(\xi)}^{L}=i \zeta \frac{\partial}{\partial \zeta} \equiv \Xi \\
& \widetilde{X}_{(q)}^{R}=\frac{\partial}{\partial q}+X_{\left(k^{j}\right)}^{R_{k} j} \Psi^{s} \frac{\partial}{\partial k^{j}}-\frac{1}{2} f_{i k}^{j} \Psi^{i} \Psi^{k} \frac{\partial}{\partial \Psi^{j}} \\
& +\Psi_{j}^{*} \frac{\partial}{\partial T_{j}}+(\lambda / 2) f_{m n}^{s} \Psi^{m} \Psi^{n} \Psi_{s}^{*} \Xi \\
& \widetilde{X}_{\left(k^{j}\right)}^{R}=X_{\left(k^{j}\right)}^{R_{k} j} \frac{\partial}{\partial k^{j}}-f_{i k}^{j} \Psi^{k} \frac{\partial}{\partial \Psi^{j}} \\
& -f_{j i}^{k} \Psi_{k}^{*} \frac{\partial}{\partial \Psi_{j}^{*}}-f_{j i}^{k} T_{k} \frac{\partial}{\partial T_{j}}, \\
& \widetilde{X}_{\left(\Psi_{i}^{*}\right)}^{R}=\frac{\partial}{\partial \Psi^{i}}-f_{j i}^{k} T_{k} \frac{\partial}{\partial \Psi_{j}^{*}}+\frac{\lambda}{2}\left(f_{i j}^{k} T_{k} \Psi^{j}+\Psi_{i}^{*}\right) \Xi, \\
& \widetilde{X}_{\left(\Psi_{i}^{*}\right)}^{R}=\frac{\partial}{\partial \Psi_{i}^{*}}+\frac{\lambda}{2} \Psi^{i} \Xi, \quad \widetilde{X}_{\left(T_{s}\right)}^{R}=\frac{\partial}{\partial T_{i}}, \\
& \widetilde{X}_{(\zeta)}^{R}=i \zeta \frac{\partial}{\partial \zeta} \equiv \Xi,
\end{aligned}
$$

where $X_{\left(k^{\prime}\right)}^{L_{k^{i}}}$ and $X_{\left(k^{\prime}\right)}^{R_{k^{i}}}$ are the components of the left- and right-invariant vector fields of the subgroup $K$.

The GAQ mechanism can now be applied just as a mathematical tool for working out the representations of $\widetilde{\mathfrak{B}}(K)$. Before going to the quantization mechanism let us compute the classical Noether invariants associated with the symmetries (4.6). Writing the one-form $\theta$ as

$$
\begin{aligned}
\Theta= & \vartheta^{L(q)} \widetilde{X}_{(q)}^{L}+\vartheta^{L(\mathrm{k})} \tilde{X}_{(\mathrm{k})}^{L}+\vartheta^{L(\Psi)} \widetilde{X}_{(\Psi)}^{L} \\
& +\vartheta^{L\left(\Psi^{*}\right)} \widetilde{X}_{\left(\Psi^{*}\right)}^{L}+\vartheta^{L(\tilde{T})} \widetilde{X}_{(\mathrm{T})}^{L}+\vartheta^{L(\xi)} \widetilde{X}_{(\xi)}^{L},
\end{aligned}
$$

the conditions $\Theta\left(\widetilde{X}_{(\zeta)}^{L}\right)=1, \Theta\left(\widetilde{X}_{\text {(any other) }}^{L}\right)=0$ lead to the very simple expression

$\Theta=\frac{\lambda}{2}\left(\Psi_{i}^{*} d \Psi^{i}+\Psi^{i} d \Psi_{i}^{*}\right)-\frac{\lambda}{2} f_{m n}^{i} \Psi^{m} \Psi^{n} d T_{i}+\frac{d \zeta}{i \zeta}$,

corresponding to some kind of multi "time" $(T)$ dynamical system with Hamilionians $H^{i}=(\lambda / 2) f_{m n}^{i} \Psi^{m} \Psi^{n}$ (note that the characteristic subalgebra is generated by $\widetilde{X}_{(q)}^{L}$ and $\left.\tilde{X}_{(T)}^{L}\right)$.

The Noether invariants are obtained at once:

$$
\begin{aligned}
& i_{\tilde{X}_{(q)}^{R}} \mathrm{O}=-(\lambda / 2) f_{m n}^{i} \Psi^{m} \Psi^{n} \Psi_{i}^{*} \equiv \unrhd, \\
& i_{\tilde{X}_{i k}^{k},} \Theta=-\lambda\left(f_{i m}^{k} \Psi^{m} \Psi_{k}^{*}-T_{n} \Psi^{n} \Psi_{i}\right) \equiv \Im_{i}, \\
& i_{\bar{X}_{\left(\psi^{\prime}\right)}^{R}} \Theta=\lambda\left(\Psi_{i}^{*}+f_{i k}^{m} \Psi^{k} T_{m}\right) \equiv \tilde{T}_{i}, \\
& i_{\hat{X}_{(\Psi *)}^{R},} \Theta=\lambda \Psi^{i} \equiv \mathfrak{F}^{* i} \\
& i_{\tilde{X}_{i, j}^{R},} \Theta=-(\lambda / 2) f_{m k}^{i} \Psi^{m} \Psi^{k} \equiv \mathfrak{T}^{i} .
\end{aligned}
$$

Among them there exist several relations and only $\tilde{f}_{i}$ and $f_{i}^{*}$ are independent. For instance, the classical BRST charge $\mathfrak{D}$ can be written for $\lambda=1$ as

$$
\mathfrak{Q}=\mathfrak{f}_{i}^{*} \mathfrak{\Im}_{i}+\frac{1}{2} f_{m n}^{i} \mathfrak{f}_{i} \mathfrak{f}^{* m} \mathrm{f}^{* n},
$$

where it must be stressed that $\mathfrak{F}$ is the total "angular momen- tum" invariant $\mathbb{Q}+\subseteq$, giving rise to the + sign in the expression for $\mathfrak{Q}^{32,33}$

Applying our quantization method to the present (super)group, the wave functions are complex functions on $\widetilde{\mathfrak{B}}(K)$ which satisfy

$$
\begin{aligned}
& \widetilde{X}_{\{\zeta)}^{R} \cdot \Psi \equiv i \zeta \frac{\partial}{\partial \xi} \Psi=i \Psi, \\
& \widetilde{X}^{L} \cdot \Psi=0, \quad \forall \widetilde{X}^{L} \in \mathscr{P} .
\end{aligned}
$$

For the particular case of $\widetilde{\mathcal{B}}(K)$ and irrespective of the dimension of $K$ (finite or infinite) there always exists a full polarization containing $\widetilde{X}_{\left(\psi_{j}^{*}\right)}^{L}, \forall j=1, \ldots, \operatorname{dim} K$,

$$
\mathscr{P}^{N}=\left\langle\widetilde{X}_{(q)}^{L}, \widetilde{X}_{\left(k^{\prime}\right)}^{L}, \widetilde{X}_{\left(\Psi_{j}^{\prime}\right)}^{L}, \widetilde{X}_{\left(T_{k}\right)}^{L}\right\rangle, \quad i, j, k=1 \ldots, \operatorname{dim} K,
$$

and this type of full polarization will be called natural polarization. ${ }^{34,22}$

Solving Eqs. (4.11) for the full polarization $\mathscr{P}^{N}$ leads to the following set of Fock states:

$$
\begin{aligned}
|1\rangle \sim \zeta W,\left|\Psi^{i}\right\rangle & \sim \zeta W \Psi^{i}, \ldots,\left|\Psi^{i_{1}} \cdots \Psi^{i_{n}}\right\rangle \\
& \sim \zeta W \Psi^{i} \ldots \Psi^{i_{n}}, \quad i_{k}=1, \ldots, \operatorname{dim} K,
\end{aligned}
$$

where the weight function $W$ is given by

$$
W=\exp (\lambda / 2) i\left(\Psi_{n}^{*} \Psi^{n}-f_{i j}^{k} \Psi^{i} \Psi^{j} T_{k}\right) .
$$

The action of the operators $\widetilde{X}^{R}$ on the basic states $|1\rangle,\left|\Psi^{i}\right\rangle$ is

$$
\begin{aligned}
& \widetilde{X}_{(q)}^{R}|1\rangle=0, \quad \bar{X}_{(q)}^{R}\left|\Psi^{i}\right\rangle=-\frac{1}{2} f_{j k}{ }^{i}\left|\Psi^{j} \Psi^{k}\right\rangle, \\
& \widetilde{X}_{\left(k^{\prime}\right)}^{k}|1\rangle=0 \text {, } \\
& \widetilde{X}_{\left(k^{\prime}\right)}^{R}\left|\Psi^{i}\right\rangle=-f_{j m}^{i}\left|\Psi^{m}\right\rangle, \quad \widetilde{X}_{\left(\psi^{k}\right)}^{R}|1\rangle=0, \\
& \widetilde{X}_{\left(\psi_{j} /\right.}^{R}\left|\Psi^{i}\right\rangle=\delta_{j}^{i}|1\rangle, \\
& \widetilde{X}_{\left(\Psi_{;}\right)}^{R}|1\rangle=i \lambda\left|\Psi^{i}\right\rangle, \quad \widetilde{X}_{\left(\Psi_{s}^{\prime}\right)}^{R}\left|\Psi^{i}\right\rangle=i \lambda\left|\Psi^{j i} \Psi^{i}\right\rangle, \\
& \widetilde{X}_{\left(T_{j}\right)}^{R}|1\rangle=-(\lambda / 2) i f_{m n}{ }^{j}\left|\Psi^{m} \Psi^{n}\right\rangle, \\
& \widetilde{X}_{\left(T_{j}\right)}^{R}\left|\Psi^{i}\right\rangle=-(\lambda / 2) i f_{m n}{ }^{j}\left|\Psi^{i} \Psi^{m} \Psi^{n}\right\rangle .
\end{aligned}
$$

The expressions for arbitrary states $\left|\Psi^{i_{1}} \cdots \Psi^{i_{n}}\right\rangle$ follow from the factorization property of wave functions. Looking at (4.14) we can find relations among operators, for instance $(\lambda=1):$

$$
i \widetilde{X}_{(q)}^{R}=\widetilde{X}_{\left(k^{\prime}\right)}^{R} \widetilde{X}_{\left(\Psi_{r}^{\prime}\right)}^{R}+\frac{1}{2} f_{m n} \widetilde{X}_{\left(\psi^{\prime}\right)}^{R} \widetilde{X}_{\left(\psi_{m, ~}^{*}\right)}^{R} \widetilde{X}_{\left(\Psi_{n}^{*}\right)}^{R} .
$$

The group $\widetilde{\mathfrak{B}}(K)$ presents a special case since, unlike for most other groups, the weight function $|1\rangle$ cannot be considered as the physical vacuum from which the creation operators generate the state space. This is due to the fact that the state $|1\rangle$ is not annihilated by all the right-invariant vector fields associated with the algebra $\mathscr{G}_{\Theta}$ (of "nonstep" operators) as is normally required, since $\widetilde{X}_{(T,}^{R}|1\rangle \neq 0$. Thus an appropriate vacuum $|0\rangle$ must be chosen from the polarized wave functions (satisfying $\widetilde{X}_{(a)}^{R}|0\rangle=0$ if the corresponding $\widetilde{X}_{(a)}^{L}$ lies in $\left.\mathscr{G}_{\theta}\right)$. When the constraint group $K$ is finite dimensional we find the "Dirac sca:"

$$
|0\rangle=\zeta W \prod_{i=1}^{\operatorname{dim} x} \Psi^{i}
$$




\section{EXAMPLES OF BRST SUPERGROUPS}

\section{A. SU(2)-BRST supergroup}

The simplest non-Abelian example of a BRST supergroup is $\widetilde{\mathfrak{R}}(\mathrm{SU}(2))$ which will play (in Sec. VI) a central role in the study of the constrained quantum free-particle. The $\widetilde{\mathfrak{B}}(\mathrm{SU}(2))$ group law can be derived at once from the general formulas (4.3) by simply specifying the matrix $R_{, j}^{i}=R_{, j}^{i}(\epsilon)$ [see (3.1)] and substituting $\eta_{. j k}^{i}$ by $f_{j k}^{i}$. It is however instructive to write down explicitly the group law for the parameter $\epsilon$ itself. The first line in the expression (4.3) suggests the ansatz

$$
\epsilon^{\prime \prime i}=\epsilon_{\mathrm{SU}(2)}^{\prime \prime i}+q^{\prime} M\left(\epsilon^{\prime}, \boldsymbol{\epsilon}\right)_{\cdot k}^{i} \Psi^{k},
$$

where $\epsilon^{\prime \prime}{ }_{S U(2)}$ is the composition law for the $S U(2)$ parameter in (3.1). In order to find $M$ we only have to compare the terms proportional to $q^{\prime}$ and antisymmetric in $i$ and $j$ in both $\left(R^{\prime} * R\right)_{j}^{i}$ and $R\left(\epsilon^{\prime \prime}\right)_{j}^{i}$. The solution for $\epsilon^{\prime \prime}$ is

$$
\begin{aligned}
\epsilon^{\mu j}= & \epsilon_{\mathrm{SU}(2)}^{\mu j}+q^{\prime} R^{-1}(\epsilon)_{k}^{m}\left[\delta_{m}^{j} \sqrt{1-\epsilon_{\mathrm{SU}(2)}^{\prime \prime} / 4}\right. \\
& \left.+\frac{1}{2} \eta_{, m l}^{i} \epsilon_{\mathrm{SU}(2)}^{\prime \prime l}\right] \Psi^{k} .
\end{aligned}
$$

We see in (5.2) (the situation in the general case is similar) how the ghosts, along with the pure BRST transformation $q$, generate ordinary rotations. A parallel phenomenon was found in the super-Poincaré group law whose supertranslations generate ordinary translations. ${ }^{35}$

The expressions for $\widetilde{X}^{L}$ and $\widetilde{X}^{R}$ are immediately obtained from the general formulas with the replacement $f_{j k}^{i} \rightarrow \eta_{. j k}^{i}$. Even more, the entire quantization (representation) process is obtained from the general one by the same replacement. Indeed, and as a consequence of the finite dimensionality, there is only one (up to equivalence) polarization which corresponds to the one described in the general case, i.e., the natural (full) polarization.

\section{B. Diff $S^{\prime}-$ BRST supergroup}

The infinite-dimensional case is in general more complicated because a full polarization leading to a physical representation may not exist. Fortunately, a nonfull polarization may provide the appropriate quantization. The nonexistence of an adequate full polarization is related to the nontrivial cohomology of the group $K$ [although the anomalous term is, of course, forbidden by the structure of the whole group $\widetilde{\mathfrak{B}}(K)]$. This is the situation for $K=\operatorname{diff} S^{1}$ whose group law is (see Ref. 4 where the general cocycle is also given):

$$
\begin{aligned}
l^{m n}= & l^{\prime n}+l^{n}+i p l^{p} l^{\prime n-p}+(i p)^{2} / 2 ! l^{p} l^{\prime m} l^{\prime n-m-p} \\
& +\cdots+\sum_{m_{1}+\ldots+m_{r}+p=n}(i p)^{r / r} / r l^{p} l^{m_{1}} \cdots l^{m_{r}}+\cdots .
\end{aligned}
$$

Using (5.3) it is not difficult to integrate an algebra of the form

$$
\begin{aligned}
& {\left[L_{n}, L_{m}\right]=i(n-m) L_{n+m},} \\
& {\left[L_{n}, \Gamma_{m}\right]=i(n-m) \Gamma_{n+m},} \\
& {\left[\Gamma_{n}, \Gamma_{m}\right]=0,}
\end{aligned}
$$

irrespective of the explicit form of the generators $\Gamma_{m}$, to get an expression for the vector realization $R_{. n}^{m}$ acting on the parameters $\gamma^{n}$ of $\Gamma_{n}$. We obtain $\gamma^{\prime \prime m}=\gamma^{\prime m}+R_{. n}^{m}\left(l^{\prime}\right) \gamma^{n}$ where

$$
\begin{aligned}
& R_{. m}^{k}(l)= \delta_{m}^{k}+\sum_{n_{1}+m=k} B_{n_{1}, m}^{k} l^{n_{1}}+\cdots \\
&+\frac{1}{j !} \sum_{n_{1}+\cdots+n_{j}+m=k} B_{n_{1} \cdots n_{j} m}^{k} l^{n_{1} \cdots} l^{n_{j}}+\cdots \\
& B_{n_{,} m}^{k} \equiv i[-n+m], \\
& B_{n_{1} n_{2}, m}^{k} \equiv i^{2} {\left[2 n_{1} n_{2}-m\left(n_{1}+n_{2}\right)+m^{2}\right], } \\
& B_{n_{1} \cdots n_{j} m}^{k} \equiv i^{j}\left[(-)^{j} j n_{1} \cdots n_{j}+(-)^{j-1}(j-1) !\right. \\
& \\
& \quad \times\left[n_{1} n_{2} \cdots n_{j-1}+n_{j} n_{1} n_{2} \cdots n_{j-2}+\cdots\right. \\
&\left.\left.\quad+n_{2} n_{3} \cdots n_{j}\right] m+\cdots+m^{j}\right] .
\end{aligned}
$$

A similar mechanism [ raising the index $m$ of $T$ in (5.4)] can be applied to determine $R^{-1}$ :

$$
\begin{aligned}
& R_{\cdot m}^{-1 k}(l)=\delta_{m}^{k}+\sum_{n_{1}+m=k} \widehat{B}_{n_{1}, m}^{k} l^{n_{1}}+\cdots \\
& \quad+\frac{1}{j !} \sum_{n_{1}+\cdots+n_{j}+m=k} \widehat{B}_{n_{1} \cdots n_{j} m}^{k} l^{n_{1}} \cdots l^{n_{j}}+\cdots, \\
& \widehat{B}_{n_{, m}}^{k} \equiv i[2 n+m], \\
& \widehat{B}_{n_{1} n_{2}, m}^{k} \equiv i^{2}\left[2 n_{1} n_{2}+2 m\left(n_{1}+n_{2}\right)+m^{2}\right], \\
& \widehat{B}_{n_{1} \cdots n_{j} m}^{k} \\
& \equiv i^{j}\left[2(-k)^{j-2}\right. \\
& \quad \times\left(n_{1} n_{2}+n_{1} n_{3}+\cdots+n_{1} n_{j}+n_{2} n_{3}\right. \\
& \left.\quad+\cdots+n_{2} n_{j}+n_{3} n_{4}+\cdots+n_{j-1} n_{j}\right) \\
& \left.\quad+2(-k)^{j-1}\left(n_{1}+n_{2}+\cdots+n_{j}\right)+(-k)^{j}\right] .
\end{aligned}
$$

As already mentioned, the structure of $\widetilde{\mathfrak{B}}(K)$ does not allow any anomalous term to appear in $K$. Thus for $K=\operatorname{diff} S^{1}$, the parameter $c$ of the conformal anomaly (central extension) in the commutator

$$
\left[\hat{L}_{n}, \hat{L}_{m}\right]=i(n-m) \hat{L}_{n+m}+\frac{1}{12}\left(c n^{3}-c^{\prime} n\right) \delta_{n,-m}
$$

must vanish. On the other hand, there are no restrictions on $c^{\prime}$, which parametrizes a family of pseudoextensions (trivial extensions behaving in the GAQ-like true extensions ). ${ }^{4}$ The commutation relations for $\widetilde{\mathfrak{B}}\left(\operatorname{diff} S^{1}\right)$ are (4.1) with $f_{m n}^{i}$ $=\sqrt{-1}(m-n) \delta_{m+n}^{i},(5.7)$ with $c=0$, and

$$
\left\{\hat{Q}, \hat{\Psi}_{n}\right\}=\hat{L}_{n}-\left(c^{\prime} / 24\right) \delta_{n, 0} \text {. }
$$

For $c^{\prime} \neq 0$ the characteristic subalgebra $\mathscr{G}_{\Theta}$ is

$$
\mathscr{G}_{\Theta}=\left\langle\widetilde{X}_{\left(l_{0}\right)}^{L}, \widetilde{X}_{\left(T_{n}\right)}^{L}\right\rangle .
$$

The natural (full) polarization $\left\langle\widetilde{X}_{(q)}^{L}, \widetilde{X}_{\left(i^{i}\right)}^{L}, \widetilde{X}_{\left(\Psi_{j}^{*}\right)}^{L}, \widetilde{X}_{\left(T_{k}\right)}^{L}\right\rangle$ has to be discarded because the corresponding (Dirac) vacuum $\zeta W \Pi_{n} \Psi^{n}$ (wedge product) is infinite and the $\widehat{L}_{0}$ operator is divergent. ${ }^{36}$ The correct quantization of $\widetilde{\mathfrak{B}}\left(\operatorname{diff} S^{1}\right)$ follows from a new kind of polarizations of "standard" type ${ }^{22}$ that are not full polarizations (they cannot contain all the generators $\left.\widetilde{X}_{\left(T_{n}\right)}^{L}, n \in \mathbb{Z}\right)$. Out of those, only the following two are not equivalent:

$$
\begin{aligned}
& \mathscr{P}^{S}=\left\langle\widetilde{X}_{\left(l^{n<0}\right)}^{L}, \widetilde{X}_{\left(\Psi^{n<0}\right)}^{L}, \widetilde{X}_{\left(\Psi_{n>0}^{*}\right)}^{L}, \widetilde{X}_{\left(T_{n>0}\right)}^{L}, \widetilde{X}_{\left(\Psi_{0}^{*}\right)}^{L}, \widetilde{X}_{(q)}^{L}\right\rangle, \\
& \mathscr{P}^{\prime S}=\left\langle\widetilde{X}_{\left(l^{n<0}\right)}^{L}, \widetilde{X}_{\left(\Psi^{n<0}\right)}^{L}, \widetilde{X}_{\left(\Psi_{n>0}^{*}\right)}^{L}, \widetilde{X}_{\left(T_{n>0}\right)}^{L}, \widetilde{X}_{\left(\psi^{0}\right)}^{L}\right\rangle .
\end{aligned}
$$


Taking (5.10a) as a polarization for the general space of wave functions one obtains a representation whose weight function (of the form $|0\rangle \sim \xi W$ where $W$ is a common factor) behaves as a vacuum invariant under (the right-polarization generated by) $\widetilde{X}_{\left(l^{n+0}\right)}^{R}, \widetilde{X}_{\left(\Psi^{n}, 0\right)}^{R}, \widetilde{X}_{\left(\Psi_{n<0}^{*}\right)}^{R}, \widetilde{X}_{\left(T_{n, 0}\right)^{\prime}}^{R}$. This is the only choice of polarization leading to a "Q-cohomology" of states in the more general scheme of the next section. We are not going to dwell on the explicit calculation of the above-mentioned representation of $\overline{\mathfrak{B}}\left(\operatorname{diff} S^{1}\right)$, since the next section deals with a bigger supergroup having $\widetilde{\mathfrak{B}}\left(\operatorname{diff} S^{1}\right)$ as its structure group and we will then have to reexamine the situation in a more physical context.

\section{ENLARGED BRST DYNAMICAL SUPERGROUP}

In the last two sections we have been concerned with the supergroup $\widetilde{\mathcal{B}}(K)$ associated with the constraint group $K$ of some unspecified physical system. In this section we shall consider the whole dynamical supergroup $\widetilde{\mathfrak{B}}(K ; \widetilde{G})$ from which the GAQ formalism determines the quantum theory of the physical system. The notation $\widetilde{\mathfrak{B}}(K ; \widetilde{G})$ stands for a supergroup enlarging $\widetilde{\mathfrak{B}}(K)$ in such a way that it contains the usual dynamical operators of type $\hat{x}, \hat{p}, \hat{H}$, etc. of a quantum group $\widetilde{G}$ of which $K$ is a subgroup. The cases $\widetilde{G}=\widetilde{\mathbb{G}}_{m}$, $K=\mathrm{SO}(3) \subset \widetilde{\mathbb{G}}_{m}$ and $\widetilde{G}=\operatorname{diff} S^{1}(S) \mathscr{L} \mathbb{R}^{1, d-1}, K=\operatorname{diff} S^{1}$ are two examples of this type that will be studied in great detail. Indeed, only concrete cases can be extensively analyzed since the procedure of enlarging $\widetilde{B}(K)$ depends very much upon the basic commutators between $\hat{x}, \hat{p}$, and $\hat{H}$. However, a common feature of the enlarged supergroups $\widetilde{\mathfrak{B}}(K ; \widetilde{G})$ is that they must contain a fermionic (bosonic) operator $\hat{\kappa}=[\hat{Q}, \hat{x}]$ associated with each bosonic (fermionic) coordinate-like operator $\hat{x}$ and a fermionic (bosonic) operator $\hat{\pi}=[\hat{Q}, \hat{p}]$ for each bosonic (fermionic) momentum-like operator $\hat{p}$. Fortunately, $[\hat{Q}, \hat{H}]$ can be kept zero so no other new algebra element is needed. These new operators are entirely analogous to the nondynamical operators $\hat{T}$ already introduced in $\widetilde{\mathfrak{B}}(K)$ and, even more, to the operators $\hat{k}$ which might be thought of as the bosonic partners of the operators $\Psi$.

$\widetilde{\mathfrak{B}}(K ; \widetilde{G})$ is a right-principal bundle $\widetilde{\mathfrak{P}}(K ; \widetilde{G})$ $\rightarrow \widetilde{\mathfrak{B}}(K ; \widetilde{G}) / \widetilde{\mathcal{B}}(K)$ with fiber $\widetilde{\mathfrak{B}}(K)$ and will be regarded as a quantum group, replacing the standard $U(1)$ phase invariance of quantum mechanics with the supersymmetry $\widetilde{B}(K)$ by means of the condition (2.19) of the general GAQ approach. The continuity equation is therefore supplemented by extra Noether currents, one of which is the BRST charge. The advantage of using this quantum supergroup rather than $\widetilde{G}$ itself, like in Sec. III, is that it automatically gives the $Q$ cohomology in the space of states, thus making easier the choice of a physical vacuum.

We now apply the GAQ formalism to the two examples mentioned above, one of which is infinite dimensional. Although the full supergroup law can be given in both cases, its explicit form is not of interest to us here. Only the expressions for the left- and right-invariant vector fields on $\widetilde{\mathfrak{B}}(K ; \widetilde{G})$ will be given. A complete group law can be obtained by using the general formulas given in Ref. 22 for which the coefficients in the components of $\widetilde{X}^{R}$ and $\widetilde{X}^{L}$ serve as an input for fixing the "gauge" freedom of choosing different parametrizations of the group.

\section{A. BRST-Supergroup-quantization of the SO(3)- constrained free-particle}

In Sec. III A we considered the central extension $\widetilde{G}_{m}$ of the Galilei group $\mathbb{G}$ and in Sec. V A the BRST supergroup associated with the $\mathrm{SO}(3)$ subgroup of $\mathbb{G}, \widetilde{B}(\mathrm{SO}(3))$. We must now find a consistent way of mixing the Lie brackets of $\widetilde{\mathfrak{B}}(\mathrm{SO}(3))$ and $\widetilde{\mathbb{G}}_{m}$. The following nonzero brackets can be added to (4.1) without violating the super-Jacobi identities:

$$
\begin{aligned}
& {\left[\hat{Q}, \hat{x}_{i}\right]=\hat{\kappa}_{i} ; \quad\left\{\hat{v}^{i}, \hat{\Psi}_{j}\right\}=-\eta_{j k}^{i} \hat{v}^{k} ;} \\
& {\left[\hat{Q}, \hat{v}_{i}\right]=\hat{v}_{i} ; \quad\left[\hat{J}_{i}, \hat{x}_{j}\right]=\eta_{i j .}{ }^{k} \hat{x}_{k} ;} \\
& {\left[\hat{x}_{i}, \hat{\mathrm{v}}^{j}\right]=-m \delta_{i}^{i} \mathbb{1} ; \quad\left[\hat{J}_{i}, \hat{v}^{j}\right]=\eta_{i, k}^{i} \hat{v}^{k} ;}
\end{aligned}
$$

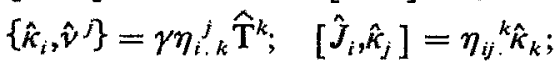

$$
\begin{aligned}
& {\left[\hat{\kappa}_{i}, \hat{v}^{j}\right]=-\gamma \eta_{i . k}{ }^{j} \Psi^{* k} ; \quad\left[\hat{J}_{i}, \hat{v}^{j}\right]=\eta_{i, k}^{j} \hat{v}^{k} ;} \\
& {\left[\hat{v}^{i}, \hat{x}_{j}\right]=\gamma \eta_{j k}^{i} \hat{\Psi}^{* k_{;}} ; \quad\left[\hat{H}, \hat{v}^{i}\right]=\delta^{i j \hat{x}_{j}} ;} \\
& \left\{\hat{\kappa}_{i}, \hat{\Psi}_{j}\right\}=-\eta_{i j}{ }^{k} \hat{x}_{k} ; \quad\left[\hat{H}, \hat{v}^{i}\right]=\delta^{i j} \hat{\kappa}_{j} ;
\end{aligned}
$$

where the constant $\gamma$ is forced to be equal to $m / \lambda$. An indication of how special the dynamical enlargement procedure leading to $\widetilde{B}\left(\operatorname{SU}(2) ; \widetilde{\mathbb{G}}_{m}\right)$ is, is the fact that the analog of the commutators $[\hat{H}, \Psi],\left[\hat{H}, \hat{\Psi}^{*}\right]$ would be nonzero for a nonfree system, for instance the harmonic oscillator.

As already mentioned, we only give here the (exact) expressions for both left- and right-invariant vector fields from which the entire quantum dynamics are derived. The left generators are

$$
\begin{aligned}
& \bar{X}_{(q)}^{L}=\frac{\partial}{\partial q}, \quad \widetilde{X}_{\left(\epsilon^{i}\right)}^{L}=X_{\left(\epsilon^{\prime}\right)}^{L \epsilon^{\prime}} \frac{\partial}{\partial \epsilon^{i}}, \\
& \bar{X}_{\left(\psi^{\prime}\right)}^{L}=R_{i}^{j} \frac{\partial}{\partial \Psi^{j}}-q X_{\left(\epsilon^{\prime}\right)}^{L \epsilon^{\prime}} \frac{\partial}{\partial \epsilon^{i}}-\frac{\lambda}{2} R^{j}{ }_{. i} \Psi_{j}^{*} \Xi, \\
& \widetilde{X}_{\left(\psi_{, j}\right)}^{L}=R-1 i\left[\frac{\partial}{\partial \Psi^{* j}}-q \frac{\partial}{\partial T_{j}}-q \eta_{s n}^{j} \Psi^{s} \frac{\partial}{\partial \Psi_{n}^{*}}\right] \\
& -(\lambda / 2) R^{-1 i} \Psi^{j} \Xi \\
& \widetilde{X}_{\left(T_{1}\right)}^{L}=R^{-1 i}\left[\frac{\partial}{\partial T_{j}}+\eta_{\cdot m n}^{j} \Psi^{m} \frac{\partial}{\partial \Psi_{n}^{*}}\right], \\
& \widetilde{X}_{\left(x^{\prime}\right)}^{L}=R_{i}^{j}\left[\frac{\partial}{\partial x^{j}}-q \frac{\partial}{\partial \kappa^{j}}-q \eta_{j m}{ }^{n} \Psi^{m} \frac{\partial}{\partial x^{n}}\right], \\
& \widetilde{X}_{\left(\kappa^{\prime}\right)}^{L}=R_{, i}^{j}\left[\frac{\partial}{\partial \kappa^{j}}+\eta_{j m}{ }^{n} \Psi^{m} \frac{\partial}{\partial x^{n}}\right] \text {, } \\
& \widetilde{X}_{\left(v_{,}\right)}^{L}=R-i i\left[\frac{\partial}{\partial v_{j}}-q \frac{\partial}{\partial v_{j}}-q \eta_{. m n}^{j} \Psi^{m} \frac{\partial}{\partial v_{n}}\right]-\gamma R-1 i, j \\
& \times \eta_{. m n}^{i} \kappa^{m} \frac{\partial}{\partial \Psi_{n}^{*}}+\gamma R^{-1 i}\left[-q \eta_{, m n}^{j}\left(\kappa^{m} \frac{\partial}{\partial T_{n}}\right.\right. \\
& \left.\left.+x^{m} \frac{\partial}{\partial \Psi_{n}^{*}}-\eta_{p r}^{n} \Psi^{m} \kappa^{p} \frac{\partial}{\partial \Psi_{r}^{*}}\right)\right]+m R \quad{ }_{j}^{1 /}\left[x^{j}\right. \\
& \left.-\frac{1}{2} \eta_{m n}^{i} \Psi^{m} \kappa^{n}-\frac{1}{2} q \eta_{\cdot m n}^{i} \Psi^{m}\left(x^{n}-\eta_{, r s}^{n} \Psi^{r} \kappa^{s}\right)\right] \Xi
\end{aligned}
$$




$$
\begin{aligned}
& \widetilde{X}_{\left(i_{1}\right)}^{\prime}=R^{-1 i},\left[\frac{\partial}{\partial v^{j}}+\eta_{. m n}^{j} \Psi^{m} \frac{\partial}{\partial v_{n}}\right]+\gamma R_{. j}^{-1 i}\left[\eta_{. m n}^{j}\right. \\
& \left.\times\left(\kappa^{m} \frac{\partial}{\partial T_{n}}+x^{m} \frac{\partial}{\partial \Psi_{n}^{*}}-\Psi^{m} \eta_{. p r}^{n} \kappa^{p} \frac{\partial}{\partial \Psi_{r}^{*}}\right)\right] \\
& +\frac{1}{2} m R-{ }^{1 i} \eta_{. m n}^{j} \Psi^{m}\left(x^{n}-\eta_{. r s}^{n} \Psi^{r} \kappa^{s}\right) \Xi, \\
& \widetilde{X}_{(t)}^{L}=\frac{\partial}{\partial t}+\delta^{i j} v_{i} \frac{\partial}{\partial x^{j}}+\delta^{i j} v_{i} \frac{\partial}{\partial \kappa^{j}}-\gamma \eta_{s}^{i k} v_{i} v_{k} \frac{\partial}{\partial \Psi_{s}^{*}} \\
& +\frac{1}{2} \gamma \eta_{k}^{i j} v_{i} v_{j} \frac{\partial}{\partial T_{k}}+m\left[\frac{1}{2} v^{2}-\frac{1}{2} \eta_{n}^{j k} v_{j} v_{k} \Psi^{n}\right] \Xi,
\end{aligned}
$$$$
\tilde{X}_{(\zeta)}^{L}=i \zeta \frac{\partial}{\partial \zeta} \equiv \Xi \text {, }
$$

where use has been made of the already known expressions $R(\epsilon)_{j}^{i}$ and $X_{\left(\epsilon^{\prime}\right)}^{L \epsilon^{\prime}}$ corresponding to the rotation subgroup [see (3.1-3.2)]. The right fields are given by

$$
\begin{aligned}
& \widetilde{X}_{(q)}^{R}=\frac{\partial}{\partial q}+X_{\left(\epsilon^{\prime}\right)}^{R \epsilon^{\prime}} \Psi^{s} \frac{\partial}{\partial \epsilon^{j}}-\frac{1}{2} \eta_{i k}^{j} \Psi^{i} \Psi^{k} \frac{\partial}{\partial \Psi^{j}} \\
& +\Psi_{j}^{*} \frac{\partial}{\partial T_{j}}-x^{i} \frac{\partial}{\partial v_{i}}+\frac{\lambda}{4} \eta_{m n}{ }^{s} \Psi^{m} \Psi^{n} \Psi_{s}^{*} \Xi, \\
& \widetilde{X}_{\left(\epsilon^{\prime}\right)}^{k}=X_{\left(\epsilon^{\prime}\right)}^{R \epsilon^{\prime}}-\eta_{i j}{ }^{k}\left(\Psi^{j} \frac{\partial}{\partial \Psi^{k}}+x^{j} \frac{\partial}{\partial x^{k}}+\kappa^{j} \frac{\partial}{\partial \kappa^{k}}\right) \\
& -\eta_{i, k}^{j}\left(\Psi_{j}^{*} \frac{\partial}{\partial \Psi_{k}^{*}}+T_{j} \frac{\partial}{\partial T_{k}}\right. \\
& \left.+v_{j} \frac{\partial}{\partial v_{k}}+v_{j} \frac{\partial}{\partial v_{k}}\right) \\
& \widetilde{X}_{\left(\Psi^{\prime}\right)}^{R}=\frac{\partial}{\partial \Psi^{\prime}}-\eta_{i, k}{ }^{j} T_{j} \frac{\partial}{\partial \Psi_{k}^{*}}+\eta_{i j .}{ }^{k} \kappa^{j} \frac{\partial}{\partial x^{k}}+\eta_{i . k}{ }^{j} v_{j} \frac{\partial}{\partial v_{k}} \\
& +(\lambda / 2)\left(\eta_{i j}{ }^{k} T_{k} \Psi^{j}+\Psi_{i}^{*}\right) \Xi \\
& \widetilde{X}_{\left(\psi_{i}^{*}\right)}^{R}=\frac{\partial}{\partial \Psi_{i}^{*}}+\frac{\lambda}{2} \Psi^{i} \Xi, \quad \widetilde{X}_{\left(T_{i}\right)}^{R}=\frac{\partial}{\partial T_{i}}, \\
& \tilde{X}_{\left(x^{\prime}\right)}^{R}=\frac{\partial}{\partial x^{i}}-\gamma \eta_{i, s}{ }^{k} v_{k} \frac{\partial}{\partial \Psi_{s}^{*}}+m\left(v_{i}-\frac{1}{2} \eta_{i m}{ }^{k} \Psi^{m} v_{k}\right) \Xi \text {, } \\
& X_{\left(u_{i}\right)}^{R}=\frac{\partial}{\partial v_{l}}+\delta^{i j_{t}} \frac{\partial}{\partial x^{j}}-\gamma t \eta_{s}^{i j} v_{j} \frac{\partial}{\partial \Psi_{s}^{*}} \\
& +m \delta^{i j} t\left(v_{j}-\frac{1}{2} \eta_{j m}{ }^{k} \Psi^{m} v_{k}\right) \Xi \text {, } \\
& \widetilde{X}_{\left(\kappa^{\prime}\right)}^{R}=\frac{\partial}{\partial \kappa^{i}}+\gamma \eta_{i, k}^{j} v_{j} \frac{\partial}{\partial \Psi_{k}^{*}} \\
& +\gamma \eta_{i, k}^{j} v_{j} \frac{\partial}{\partial T_{k}}-\frac{1}{2} m \eta_{i m}{ }^{j} \Psi^{m} v_{j} \Xi \\
& \bar{X}_{\left(v_{1}\right)}^{R}=\frac{\partial}{\partial v_{i}}+\delta^{i j} t \frac{\partial}{\partial \kappa^{j}}+\gamma t \eta_{s}^{i j} v_{j} \frac{\partial}{\partial \Psi_{s}^{*}}+\gamma t \eta_{s}^{i j} v_{j} \frac{\partial}{\partial T_{s}} \\
& -\frac{1}{2} m t \eta_{m}^{\prime \prime} \Psi^{m} v_{j} \Xi \text {, } \\
& \widetilde{X}_{(t)}^{R}=\frac{\partial}{\partial t}, \quad \widetilde{X}_{(\zeta)}^{R}=i \zeta \frac{\partial}{\partial \zeta} \equiv \Xi .
\end{aligned}
$$

The brackets (4.1), (6.1), and the omitted vanishing ones are automatically satisfied by (6.3) and the same brackets but for a global minus sign by (6.2). Another crucial condition that (6.2) and (6.3) must verify in order to be the leftand right-generators of a group law is $\left[\widetilde{X}^{L}, \widetilde{X}^{R}\right]=0$, which in fact holds.

The quantization form $\Theta$ is calculated by using duality on (6.2) and obviously generalizes (2.12) and (4.8):

$$
\begin{aligned}
\Theta= & -m(\mathbf{x}-\Psi \times \boldsymbol{K}) \cdot d \mathbf{v}-\frac{1}{2} m(\mathbf{v}-\boldsymbol{v} \times \boldsymbol{\Psi})^{2} d t \\
& +(\lambda / 2)\left(\Psi^{*} \cdot d \Psi+\Psi \cdot d \Psi^{*}\right)-\frac{\lambda}{2}(\Psi \times \Psi) \cdot d \mathbf{T} \\
& +m[\Psi \times \mathbf{X}+(\Psi \cdot \mathbf{\Psi}) \Psi] \cdot d \boldsymbol{v}+\frac{d \zeta}{i \zeta} .
\end{aligned}
$$

The Noether invariants are the inner products $i_{\tilde{X}^{R}} \Theta$ where $\widetilde{X}^{R}$ runs over the whole right algebra. They also generalize (2.13) and (4.9) for the case $K=\mathrm{SO}(3)$ :

$$
\begin{aligned}
& i_{\tilde{X}_{(q)}^{R}} \Theta=-(\lambda / 2)(\Psi \times \Psi) \cdot \Psi^{*}-m[\Psi \times \mathbf{x} \\
& -\Psi \times(\Psi \times \kappa)] \cdot \mathbf{v} \equiv \mathfrak{Q}, \\
& i_{\tilde{X}_{(\epsilon)}^{R}} \Theta=-\lambda\left[\Psi^{*} \times \Psi+(\mathbf{T} \cdot \Psi) \Psi\right]-m(\mathbf{x}-\Psi \times \boldsymbol{\Psi}) \times \mathbf{v} \\
& -m \boldsymbol{v} \times[\boldsymbol{\Psi} \times \mathbf{x}+\Psi \times(\Psi \times \kappa)] \equiv \mathfrak{J}, \\
& i_{\hat{X}_{(\Psi)}^{R}} \Theta=\lambda\left(\Psi^{*}-\mathbf{T} \times \Psi\right)-m v \times(\mathbf{x}-\Psi \times \mathbf{k}) \equiv \mathfrak{f}, \\
& i_{\widetilde{X}_{(\Psi))}^{R}} \Theta=\lambda \Psi \equiv \mathfrak{f}^{*}, \quad i_{\widetilde{X}_{(\mathfrak{T})}^{R}} \Theta=-(\lambda / 2) \Psi \times \Psi \equiv \mathfrak{T}, \\
& i_{\tilde{X}_{(\mathrm{x})}^{R}} \Theta=m(\mathbf{v}-\boldsymbol{v} \times \Psi) \equiv \mathfrak{E}, \\
& i_{\hat{X}_{(v)}^{R}} \Theta=-m(\mathbf{x}-\boldsymbol{\Psi} \times \boldsymbol{k})+m t(\mathbf{v}-\boldsymbol{v} \times \mathbf{\Psi}) \equiv \mathfrak{v}, \\
& i_{\tilde{X}_{(\mathcal{K})}^{R}} \Theta=m(\mathbf{v}-\boldsymbol{v} \times \Psi) \times \Psi=\mathbf{\Psi}, \\
& i_{\widetilde{X}_{(v)}^{R}} \Theta=-m(\mathbf{x}-\Psi \times \mathbf{\Psi}) \times \Psi+m t(\mathbf{v}-\boldsymbol{v} \times \Psi) \times \Psi \equiv \mathfrak{n}, \\
& i_{\widetilde{X}_{(i)}^{R}} \boldsymbol{\theta}=-\frac{1}{2} m(\mathbf{v}-\boldsymbol{v} \times \Psi)^{2} \equiv \mathfrak{h} .
\end{aligned}
$$

All of them can be written in terms of the basic ones $\mathfrak{x}, \mathfrak{p}, \mathfrak{f}, \mathfrak{f}^{*}$, and, in particular, $\mathfrak{Q}$ assumes the standard form (4.10).

The characteristic subalgebra, which is generated by left-vector fields in the kernel of both $\Theta$ and $d \theta$, is given by

$$
\mathscr{F}_{\Theta}=\left\langle\widetilde{X}_{(q)}^{L}, \widetilde{X}_{(\mathbf{\epsilon})}^{L}, \widetilde{X}_{(\mathbf{T})}^{L}, \widetilde{X}_{(\boldsymbol{\kappa})}^{L}, \widetilde{X}_{(\boldsymbol{v})}^{L}, \widetilde{X}_{(t)}^{L}\right\rangle
$$

and the following full polarization can be chosen:

$$
\mathscr{P}=\left\langle\mathscr{G}_{\Theta}, \widetilde{X}_{\left(\boldsymbol{\Psi}^{*}\right)}^{L}, \widetilde{X}_{(\mathbf{x})}^{L}\right\rangle .
$$

According to the general scheme of Sec. II the physical wave functions satisfy $(2.18)$ with the polarization given in (6.7) and (2.19) with $T=\widetilde{\mathfrak{B}}(\mathrm{SU}(2))$. We first solve the polarization conditions and (2.19) for just the $U(1)$ generator of $\tilde{\mathrm{b}}(\mathrm{SU}(2))\left(\widetilde{X}_{(\zeta)}^{R} \cdot \Psi=i \Psi\right)$ and then the rest of (2.19) (in this way we can see what would have been the unconstrained wave functions associated with the fibration $\widetilde{\mathfrak{B}}\left(\mathrm{SU}(2) ; \widetilde{\mathbb{G}}_{m}\right) \rightarrow \widetilde{\mathfrak{B}}\left(\mathrm{SU}(2) ; \widetilde{\mathbb{G}}_{m}\right) / \mathrm{U}(1)$. These equations lead to

$$
\begin{aligned}
\psi= & \sum C_{i_{1} \cdots i_{r}} \zeta \Phi(\mathbf{v}-\Psi \times v) \exp \left[-i / 2(\mathbf{v}-\Psi \times v)^{2} m t\right] \\
& \times \exp (i(\lambda / 2)[\Psi * \cdot \Psi-(\Psi \times \Psi) \cdot \mathbf{T}]) \\
& \times \Psi^{i_{1}} \Psi^{i_{2}} \cdots \Psi^{i_{r}}, \quad r=1,2,3
\end{aligned}
$$

where $\Phi$ is an arbitrary function of $\mathbf{v}-\Psi \times v$ and $C_{i_{1} i_{2} \cdots i_{r}}$ are arbitrary constants. The remaining equations force $C_{i_{1} i_{2} \cdots i_{r}}$ to be zero, apart from $C_{123}$ which remains free. Developing $\psi$ 
in a power series, only the leading term contributes since the "Dirac sea" $\Psi^{1} \Psi^{2} \Psi^{3}$ in (6.8) does not allow for any additional power of $\Psi$.

Therefore, the final wave functions are

$$
\psi=\xi \varphi(\mathrm{v}) e^{-i(m / 2) \mathrm{v}^{2} t} \Psi^{1} \Psi^{2} \Psi^{3},
$$

where the function $\varphi$ satisfies either (3.6) or (3.7) according to the particular representation of $\mathrm{SU}(2)$ chosen in $(2.19)$.

\section{B. BRST supergroup quantization of the free-string}

In this example, $\widetilde{\mathfrak{B}}\left(\operatorname{diff} S^{1}\right)$ must be enlarged so as to incorporate the transformations generated by the operators of type $\hat{x}, \hat{p}$ for the string. They are the generators of the loop group in $\mathbb{R}^{1, d-1}, \hat{\alpha}_{n}^{\mu}, \mu=0, \ldots 25, n \in \mathbb{Z}$. The energy operator $\hat{L}_{0} \equiv H$ is already contained in the constraint subgroup $K \equiv$ diff $S^{1}$. An admissible Lie superalgebra for the complete supersymmetry has the following nonzero brackets:

$$
\begin{aligned}
& {\left[\hat{\alpha}_{n}^{\mu}, \hat{\alpha}_{m}^{v}\right]=n g^{\mu v} \delta_{n,-m} \hat{\mathbf{1}} \equiv \Sigma_{n m}^{\mu v} \hat{\mathbf{1}} \text {; }} \\
& {\left[\hat{L}_{n}, \hat{L}_{m}\right]=(n-m) \hat{L}_{n+m}-c^{\prime} / 12 n \delta_{n,-m}} \\
& =f_{n m}{ }^{k} \hat{L}_{k}-c^{\prime} / 12 n \delta_{n,-m}, \\
& {\left[\hat{L}_{n}, \hat{\alpha}_{m}^{\mu}\right]=m \hat{\alpha}_{n+m}^{\mu} \equiv F_{n m}{ }^{k} \hat{\alpha}_{k}^{\mu} ; \quad\left\{\hat{\Psi}_{n}, \hat{\Psi}^{* m}\right\}=\lambda \delta_{n}^{m} \hat{\mathbf{1}},} \\
& {\left[\hat{L}_{n}, \hat{\Psi}_{\mu}\right]=(n-m) \hat{\Psi}_{n+m} \equiv f_{n m}{ }^{k} \hat{\Psi}_{k} \text {, }} \\
& {\left[\hat{L}_{n}, \hat{\Psi}_{m}^{*}\right]=-(2 n+m) \hat{\Psi}^{* n+m} \equiv-f_{n k} m \hat{\Psi}^{* k} \text {, }} \\
& {\left[\hat{Q}, \hat{\alpha}_{n}^{\mu}\right]=\hat{\pi}_{n}^{\mu} ; \quad\left\{\hat{Q}, \hat{\Psi}_{n}\right\}=\hat{L}_{n}-c^{\prime} / 24 \delta_{n, o} \hat{\mathbb{1}}} \\
& \left\{\hat{Q}, \hat{\Psi}^{*}{ }_{n}\right\}=\widehat{T}_{n} \text {, } \\
& {\left[\hat{L}_{n}, \hat{\pi}_{m}^{\mu}\right]=m \hat{\pi}_{n+m}^{\mu} \equiv F_{n m}{ }^{k} \hat{\pi}_{k}^{\mu} \text {; }} \\
& {\left[\hat{L}_{n}, \hat{T}^{m}\right]=-(2 n+m) \hat{T}^{n+m} \equiv-f_{n k}{ }^{m} \hat{T}^{k} \text {, }} \\
& {\left[\hat{\pi}_{n}^{\mu}, \hat{\alpha}_{m}^{v}\right]=-\gamma n m g^{\mu v} \hat{\Psi}^{* n+m} \equiv-\gamma \Sigma_{n k}^{\mu v} F_{m s}{ }^{k} \hat{\Psi}^{* s} \text {, }} \\
& \left\{\hat{\pi}_{n}^{\mu}, \hat{\pi}_{m}^{v}\right\}=\gamma n m g^{\mu v} \widehat{T}^{n+m} \equiv \gamma \Sigma_{n k}^{\mu \nu} F_{m s}{ }^{k} \widehat{T}^{s} \text {, } \\
& \left\{\hat{\pi}_{n}^{\mu}, \hat{\Psi}_{m}\right\}=m \hat{\alpha}_{n+m}^{\mu} \equiv F_{n m}{ }^{k} \hat{\alpha}_{k}^{\mu} ; \quad\left[\hat{\Psi}_{n}, \hat{T}^{m}\right]=-f_{n k}{ }^{m} \hat{\Psi}^{* k} \text {, }
\end{aligned}
$$

where the constant $\gamma$ is again fixed to $1 / \lambda$ (the central extension parameter for the $\hat{\alpha}$ 's has been put equal to 1 ).

The search for an exact expression for both left- and right invariant vector fields reproducing (6.10) and the same formulas but for a minus sign can be undertaken following the general method of Ref. 22 , as we have done indeed for the subgroup diff $S^{1}$ whose exact formal group law can be found in Ref. 4. However, if we are only interested in the expressions for the left and right algebras, it is far easier to take advantage of the experience acquired in the previous example and the already known exact expressions for diff $S^{1}$. We write directly the left fields (keeping the general constants $f_{m n}^{k}, F_{s t}^{r}$, and $\Sigma_{p q}^{\mu v}$ facilitates the calculations):

$$
\begin{aligned}
& \widetilde{X}_{(q)}^{L}=\frac{\partial}{\partial q}+X_{\left(l^{n}\right)}^{L l^{m}} \Psi^{n} \frac{\partial}{\partial l^{m}}-\frac{1}{2} f_{r s}^{m} \Psi^{r} \Psi^{s} \frac{\partial}{\partial \Psi^{m}} \\
& +\Psi_{m}^{*} \frac{\partial}{\partial T_{m}}-\alpha_{\mu}^{m} \frac{\partial}{\partial \pi_{\mu}^{m}}+\frac{\lambda}{4} f_{m n}^{s} \Psi^{m} \Psi^{m} \Psi_{s}^{*} \Xi \\
& +\frac{c^{\prime}}{24} X_{\left(l^{n}\right)}^{L l^{0}} \Psi^{n} \Xi \\
& \widetilde{X}_{\left(l^{\prime}\right)}^{L}=X_{\left(l^{\prime}\right)}^{L l^{m}} \frac{\partial}{\partial l^{m}}-f_{i n}^{m} \Psi^{n} \frac{\partial}{\partial \Psi^{m}}+f_{i n}^{m}\left(\Psi_{m}^{*} \frac{\partial}{\partial \Psi_{n}^{*}}\right. \\
& \left.+T_{m} \frac{\partial}{\partial T_{n}}\right)+F_{i n}^{m}\left(\alpha_{\mu}^{n} \frac{\partial}{\partial \alpha_{\mu}^{m}}+\pi_{\mu}^{n} \frac{\partial}{\partial \pi_{\mu}^{m}}\right) \\
& +\frac{c^{\prime}}{24}\left(X_{\left(l^{\prime}\right)}^{L l^{9}}-\delta_{i, 0}\right) \Xi \\
& \widetilde{X}_{\left(\psi^{i}\right)}^{L}=\frac{\partial}{\partial \Psi^{i}}+f_{i k}^{j} T_{j} \frac{\partial}{\partial \Psi_{k}^{*}}+F_{i k}^{j} \pi_{\mu}^{k} \frac{\partial}{\partial \alpha_{\mu}^{j}} \\
& +\frac{\lambda}{2}\left(f_{i j}^{k} T_{k}^{j} \Psi^{*}+\Psi_{i}\right) \Xi \\
& \widetilde{X}_{\left(\Psi_{i}^{*}\right)}^{L}=\frac{\partial}{\partial \Psi^{*}{ }_{i}}+\frac{\lambda}{2} \Psi^{i} \Xi, \quad \widetilde{X}_{\left(T_{i}\right)}^{L}=\frac{\partial}{\partial T_{i}}, \\
& \widetilde{X}_{\left(\alpha_{\mu}^{n}\right)}^{L}=\frac{\partial}{\partial \alpha_{\mu}^{n}}+\frac{\gamma}{2} \Sigma_{s r}^{\mu v} F_{k n}^{s} \pi_{v}^{r} \frac{\partial}{\partial \Psi_{k}^{*}}-\frac{\kappa}{2} \Sigma_{n s}^{\mu v}\left(\alpha_{v}^{s}\right. \\
& \left.-\frac{1}{2} F_{m p}^{s} \Psi^{m} \pi_{v}^{p}\right) \Xi \\
& \widetilde{X}_{\left(\pi_{\mu}^{*}\right\rangle}^{L}=\frac{\partial}{\partial \pi_{\mu}^{n}}-\frac{\gamma}{2} \Sigma_{s r}^{\mu v} F_{k n}^{s} \alpha_{r}^{r} \frac{\partial}{\partial \Psi_{k}^{*}}-\frac{\gamma}{2} \Sigma_{s r}^{\mu v} F_{k n}^{s} \pi_{v}^{r} \frac{\partial}{\partial T_{k}} \\
& +(\kappa / 2) \Sigma_{n s}^{\mu v} F_{m p}^{s} \Psi^{m} \alpha_{v}^{p} \Xi \text {, } \\
& \bar{X}_{(\xi)}^{L}=i \xi \frac{\partial}{\partial \zeta} \equiv \Xi
\end{aligned}
$$

where the components of the (unextended) diff $S^{1}$ left generators are

$$
X_{\left(i^{*}\right)}^{L l^{m}}=\delta_{n}^{m}+i(m-n) l^{m-n}
$$

For the right-invariant vector fields we find:

$$
\begin{aligned}
& \widetilde{X}_{(q)}^{R}=\frac{\partial}{\partial q}, \quad \widetilde{X}_{\left(l^{\prime \prime}\right)}^{R}=X_{\left(l^{n}\right)}^{R l^{m}} \frac{\partial}{\partial l^{m}}+\frac{c^{\prime}}{24}\left(X_{\left(l^{\prime \prime}\right)}^{R l^{0}}-\delta_{n, 0}\right) \Xi, \\
& \widetilde{X}_{\left(\Psi^{m}\right)}^{R}=R_{\cdot n}^{m} \frac{\partial}{\partial \Psi^{m}}+q X_{\left(l^{n}\right)}^{R l^{m}} \frac{\partial}{\partial l^{m}}-\frac{\lambda}{2} R_{\cdot n}^{m} \Psi_{m}^{*} \Xi-\frac{c^{\prime}}{24} q X_{\left(l^{\prime \prime}\right)}^{R l^{*}} \Xi, \\
& \widetilde{X}_{\left(\Psi_{n}^{*}\right)}^{R}=\left(R^{-1}\right)_{\cdot m}^{n}\left(\frac{\partial}{\partial \Psi_{m}^{*}}-q \frac{\partial}{\partial T_{m}}-f_{s n}^{m} \Psi^{s} \frac{\partial}{\partial \Psi_{n}^{*}}\right)-\frac{\lambda}{2}\left(R^{-1}\right)_{\cdot m}^{n} \Psi^{m} \Xi, \\
& \widetilde{X}_{\left(T_{n}\right)}^{R}=\left(R^{-1}\right)_{\cdot m}^{n}\left(\frac{\partial}{\partial T_{m}}+f_{s n}^{m} \Psi^{s} \frac{\partial}{\partial \Psi_{n}^{*}}\right),
\end{aligned}
$$




$$
\begin{aligned}
& \widetilde{X}_{\left(\alpha_{\mu}^{n}\right)}^{R}=U_{\cdot n}^{m}\left(\frac{\partial}{\partial \alpha_{\mu}^{m}}-q \frac{\partial}{\partial \pi_{\mu}^{m}}+q F_{s m}^{p} \Psi^{s} \frac{\partial}{\partial \alpha_{\mu}^{p}}\right)+\frac{\gamma}{2} U_{. n}^{m} \Sigma_{m s}^{\mu v} F_{p r}^{s} \pi_{v}^{r} \frac{\partial}{\partial \Psi_{p}^{*}} \\
& +\frac{\gamma}{2} U_{. n}^{m} \Sigma_{m s}^{\mu v} F_{p r}^{s} q\left(\pi_{v}^{r} \frac{\partial}{\partial T_{p}}+\alpha_{v}^{r} \frac{\partial}{\partial \Psi_{p}^{*}}\right)+\frac{\gamma}{2} U_{. n}^{m} q \Sigma_{s p}^{\mu \nu} F_{r t}^{p} F_{v m}^{s} \Psi^{v} \pi_{v}^{t} \frac{\partial}{\partial \Psi_{r}^{*}} \\
& +\frac{\kappa}{2} U_{. n}^{m}\left[\Sigma_{m s}^{\mu \nu}\left(\alpha_{v}^{s}-\frac{1}{2} F_{r p}^{s} \Psi^{r} \pi_{v}^{p}-\frac{1}{2} q F_{r p}^{s} \Psi^{r} \alpha_{v}^{p}\right)-\frac{1}{2} q \Sigma_{s p}^{\mu \nu} F_{t r}^{p} F_{v m}^{s} \Psi^{\prime} \Psi^{v} \pi_{v}^{r}\right] \Xi, \\
& \widetilde{X}_{\left(\pi_{\mu}^{\prime \prime}\right)}^{R}=U_{. n}^{m}\left(\frac{\partial}{\partial \pi_{\mu}^{m}}-F_{s m}^{p} \Psi^{s} \frac{\partial}{\partial \alpha_{\mu}^{p}}\right)-\frac{\gamma}{2} U_{. n}^{m} \Sigma_{m s}^{\mu v} F_{p r}^{s}\left(\pi_{v}^{r} \frac{\partial}{\partial T_{p}}+\alpha_{v}^{r} \frac{\partial}{\partial \Psi_{p}^{*}}\right) \\
& -\frac{\gamma}{2} U_{. n}^{m} \Sigma_{s p}^{\mu v} F_{r t}^{p} F_{v m}^{s} \Psi^{v} \pi_{v}^{t} \frac{\partial}{\partial \Psi_{r}^{*}}+\frac{\kappa}{4} U_{. n}^{m}\left(\Sigma_{m s}^{\mu \nu} F_{r p}^{s} \Psi^{r} \alpha_{v}^{p}+\Sigma_{s p}^{\mu v} F_{t r}^{p} F_{v m}^{s} \Psi^{t} \Psi^{v} \pi_{v}^{r}\right) \Xi, \\
& \widetilde{X}_{(\zeta)}^{R}=i \zeta \frac{\partial}{\partial \zeta} \equiv \Xi \text {, }
\end{aligned}
$$

which commute with (6.11) $)^{37}$ (See Ref. 4 for expression of the matrix $u$.)

We shall bypass the explicit calculation of the quantization form $\theta$ and guess the characteristic subalgebra $\mathscr{G}_{\theta}$ directly from the structure constants. The form $\Theta$ could be exactly computed in a straightforward way and the Noether invariants derived, but nothing essentially new would appear with respect to the former example. Hence, we give the characteristic subalgebra:

$$
\mathscr{G}_{\Theta}=\left\langle\widetilde{X}_{(q)}^{L}, \widetilde{X}_{\left(l^{0}\right)}^{L}, \widetilde{X}_{\left(T_{m}\right)}^{L}, \widetilde{X}_{\left(\pi_{\mu}^{k}\right)}^{L}\right\rangle .
$$

As in the case of $\widetilde{\mathfrak{B}}\left(\operatorname{diff} S^{1}\right)$ no full polarization exists that leads to a vacuum for which the $L_{0}$ operator is finite. Hence, we must resort to a nonfull polarization containing only a subalgebra $\mathscr{A}^{L}$ of $\mathscr{G}_{\Theta}$. This is related to the existence of anomalies and could in fact be used as their characterization rather than the appearance of central terms in the quantum commutators. Indeed a (super)group like $\widetilde{B}$ (diff $S^{1}$; diff $S$ (S) $\mathscr{L} \mathbf{R}^{1, d-1}$ ) does not allow for such a central term in the Lie algebra of $\operatorname{diff} S^{1}$. However, the physical anomaly manifests itself when writing down the operators $\left(\widetilde{X}^{R}\right)$, which turn out to be functions of more than the basic operators, i.e., those involved in the commutators producing central terms (all operators are expressed in terms of the basic operators as well as those operators whose left counterparts are in $\mathscr{G}_{\Theta}$ although not in $\mathscr{A}^{L}$ ). Only for critical values of the constant parameters characterizing the whole algebra all operators $\widetilde{X}^{R}$ can be written in terms of the basic operators (in our case the $\hat{\alpha}$ 's, $\hat{\Psi}^{*}$ 's, and $\hat{\Psi}$ 's; see later).

The only (nonfull) polarization leading to the $Q$ cohomology (and to unitary representations) is the following one:

$$
\begin{gathered}
\mathscr{P}^{S}=\left\langle\widetilde{X}_{(q)}^{L}, \widetilde{X}_{\left(l^{n<0}\right)}^{L}, \widetilde{X}_{\left(T_{n>0}\right)}^{L}, \widetilde{X}_{\left(\psi_{n>0}^{*}\right)}^{L},\right. \\
\left.\tilde{X}_{\left(\Psi^{n<0}\right)}^{L}, \widetilde{X}_{\left(\alpha_{\mu}^{n<0}\right)}^{L}, \widetilde{X}_{\left(\pi_{v}^{n<0}\right)}^{L}\right\rangle .
\end{gathered}
$$

Equation (6.15) is of standard type and contains (5.10a). The integration of the polarization cquations $\widetilde{X}^{L} \Psi=0 \forall \widetilde{X}^{L} \in \mathscr{P} S$ will be given to third order in the group parameters (of course all main results do appear at this level). The wave functions are $|0\rangle \sim \zeta W_{1}(q, l, \Psi) W_{2}\left(\Psi, \Psi^{*}, T, \alpha, \pi, l\right) \equiv \zeta W$,

$$
\begin{aligned}
& \left|l^{i>0}\right\rangle \sim \zeta W\left\{l^{i}-\sum_{k>0} k l^{k} l^{i-k}-q \Psi^{i}+\cdots\right\}, \\
& \left|T_{i<0}\right\rangle \sim \zeta W\left\{T_{i}-\sum_{k<0} f_{m i}^{k} l^{m} T_{k}\right. \\
& \left.+\frac{1}{2} \gamma \sum_{k<0} \Sigma_{s k}^{\mu \nu} F_{i m}^{s} \pi_{\mu}^{k} \pi_{v}^{m}-q \Psi_{i}^{*}+\cdots\right\},
\end{aligned}
$$$$
\left|\pi_{\mu}^{n>0}\right\rangle \sim \zeta W\left\{\pi_{\mu}^{n}-\sum_{k>0} F_{m k}^{n} l^{m} \pi_{\mu}^{k}+q \alpha_{\mu}^{n}+\cdots\right\},
$$$$
\left|\alpha_{\mu}^{n>0}\right\rangle \sim \zeta W\left\{\alpha_{\mu}^{n}-\sum_{k>0} F_{m k}^{n} l^{m} \alpha_{\mu}^{k}\right.
$$$$
\left.-\sum_{m<0} F_{m k}^{n} \Psi^{m} \pi_{\mu}^{k}+\cdots\right\} \text {, }
$$

$$
\left|\Psi^{i>0}\right\rangle \sim \zeta W\left\{\Psi^{i}+\sum_{k>0} f_{m k}^{i} l^{m} \Psi^{k}+\cdots\right\}
$$$$
\left|\Psi_{i<0}^{*}\right\rangle \sim \zeta W\left\{\Psi_{i}^{*}-\sum_{k<0} f_{m i}^{k} l^{m} \Psi_{k}^{*}-\sum_{k<0} f_{m i}^{k} T_{k} \Psi^{m}\right.
$$

$$
-\frac{1}{2} \gamma \sum_{k>0} \Sigma_{s k}^{\mu \nu} F_{i m}^{s} \pi_{\mu}^{k} \alpha_{v}^{m}
$$$$
\left.+\frac{1}{2} \gamma \sum_{k>0} \Sigma_{s k}^{\mu \nu} F_{i m}^{s} \alpha_{\mu}^{k} \pi_{v}^{m}+\cdots\right\},
$$

$$
\begin{aligned}
& \left|\left(l^{i_{1}}\right)^{n_{i_{1}}}\left(l^{i_{2}}\right)^{n_{i_{2}}} \cdots\left(T_{j 1}\right)^{n_{j_{1}}} \cdot\left(T_{j 2}\right)^{n_{j_{2}}} \cdots\right\rangle \\
& \quad=\zeta W\left\{l^{i_{1}}+\cdots\right\}^{n_{i_{1}}}\left\{l^{i_{2}}+\cdots\right\}^{n_{i_{2}}} \cdots\left\{T_{j_{1}}+\cdots\right\}^{n_{j_{1}}} \cdots,
\end{aligned}
$$

where the factors $W_{1}$ and $W_{2}$ of the weight function associated with the vacuum are the following 


$$
\begin{aligned}
& W_{2}=1-\frac{\lambda}{2} i\left(\sum_{n>0} \Psi_{n}^{*} \Psi^{n}-\sum_{n<0} \Psi_{n}^{*} \Psi^{n}-\sum_{n, m, k<0} f_{n m}^{k}\right. \\
& \left.\times \Psi^{n} \Psi^{m} T_{k}+2 \sum_{\substack{n>0 \\
m, k<0}} f_{n k}^{m} \Psi_{m}^{*} \Psi^{n} l^{k}+\cdots\right) \\
& -\frac{\kappa}{2} i\left(\sum_{n \geqslant 0} \Sigma_{n k}^{\mu v} \alpha_{\mu}^{n} \alpha_{v}^{k}-\sum_{\substack{n \geqslant 0 \\
m, k<0}} F_{k m}^{j} \Sigma_{m j}^{\mu v} \alpha_{\mu}^{n} \pi_{v}^{m} \Psi^{k}\right. \\
& \left.-\sum_{\substack{n<0 \\
k, m>0}} F_{k m}{ }^{j} \Sigma_{n j}^{\mu v} \alpha_{\mu}^{n} \alpha_{v}^{m} l^{k}+\cdots\right), \\
& W_{1}=\tilde{W}_{1}(l)-\frac{i c^{\prime}}{24} q \sum_{n>0} \Psi^{n}\left[n \left(-l^{-n}+2 \sum_{m>0}\right.\right. \\
& \left.\times m l^{m} l^{-n-m}-3 \sum_{m, k>0} m k l^{m} l^{k} l^{-n-m-k}+\cdots\right) \\
& +\sum_{m>0}(-m-n) l^{-m-n}\left(-m l^{m}\right. \\
& \left.\left.+\sum_{\substack{j, s>0 \\
j+s=m}} j s l^{j} l^{s}-\sum_{\substack{j, s, r>0 \\
j+s+r=m}} j s r l^{j} l^{s} l^{r}+\cdots\right)\right], \\
& \tilde{W}_{1}(l)=1+\frac{i c^{\prime}}{24}\left[\sum_{n>0}-n l^{n} l^{-n}+\sum_{n, m>0}\right. \\
& \times n m l^{n} l^{m} l^{-n-m}-\sum_{n, m, k>0} \\
& \left.\times n m k l^{n} l^{m} l^{k} l^{-n-m-k}+\cdots\right] .
\end{aligned}
$$

Here, $\widetilde{W}_{1}(l)$ is just the weight function of the Virasoro subgroup itself and $W_{1}(l, q, \Psi)$ is the weight function that would correspond to the Bowick-Gürsey subalgebra ${ }^{32}$ (here generated by $\left.\widetilde{X}_{\left(l^{n}\right)}, \widetilde{X}_{\left(\psi^{m}\right)}, \widetilde{X}_{(q)}\right)$.

The physical operators, the right-invariant vector fields, are well-defined differential operators acting on (6.16). We will not write the explicit action of all of them, limiting ourselves to those calculations that are strictly necessary for determining the critical values of $c^{\prime}$ and $d$ (the space-time dimension). The action of $\widetilde{X}_{\left(l^{k-9}\right)}^{R}, \bar{X}_{\left(\alpha_{k i}^{n-0}\right)}^{R}$ and $\widetilde{X}_{\left(\psi_{m, 0}^{*}\right)}^{R}$ on $|0\rangle$ and that of $\widetilde{X}_{\left(\alpha_{1}^{n},\right.}^{R}$ on $\left|\alpha_{\mu}^{m}\right\rangle$ and $\widetilde{X}_{\left(\psi^{\prime \prime}\right)}^{R}$ on $\left|\Psi^{k}\right\rangle$ are given by

$$
\begin{aligned}
& \widetilde{X}_{\left(l^{-n \cdot 3)}\right.}^{R}|0\rangle=\frac{-i c^{\prime}}{12} n\left|l^{n}\right\rangle+\frac{i \kappa}{2} F_{-n m}^{s} \Sigma_{r s}^{\mu \nu}\left|\alpha_{\mu}^{r} \alpha_{\nu}^{m}\right\rangle \\
& -i \lambda f_{r-n}^{m}\left|\Psi_{m}^{*} \Psi^{r}\right\rangle+\cdots \\
& =\frac{-i c^{\prime}}{12} n\left|l^{n}\right\rangle+\frac{i \kappa}{2} g^{u v} \sum_{0<m<n} \\
& \times m(n-m)\left|\alpha_{\mu}^{n-m} \alpha_{v}^{m}\right\rangle-i \lambda \sum_{0<r<n} \\
& \times(r+n)\left|\Psi_{r-n}^{*} \Psi^{r}\right\rangle+\cdots, \\
& \tilde{X}_{\left(\alpha_{\mu}^{\prime m-\alpha},\right.}^{R}|0\rangle=-i \kappa \Sigma_{n m}^{\mu \nu}\left|\alpha_{v}^{n}\right\rangle+\cdots, \\
& \widetilde{X}_{\left(\Psi_{m+0}^{*}\right)}^{R}|0\rangle=-i \lambda\left|\Psi^{m}\right\rangle+\cdots, \\
& \widetilde{X}_{\left(\alpha_{1}^{r}, 0,0\right.}^{R}\left|\alpha_{\mu}^{n}\right\rangle=-i \kappa \Sigma_{p r}^{v \rho}\left|\alpha_{\rho}^{p} \alpha_{\mu}^{n}\right\rangle+r \delta_{\mu}^{\nu}\left|l^{n-r}\right\rangle+\cdots,
\end{aligned}
$$

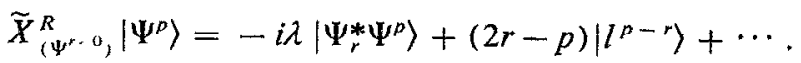

The representation obtained in this way is, in general, reducible. The reduction can then be achieved by choosing a vacuum according to the prescription of Sec. II (the weight function in the present case) from which the nontrivial action of the operators generate the (irreducible) Fock space. It must be stressed that this construction is rather different from the ordinary abstract one of the Lie algebra representation. There, all the states are obtained from the vacuum through the action of the creation operators which are, by definition, linearly independent. On the contrary, our states, in particular the vacuum, are explicit functions on the (super) group whose linear dependence or independence is prior to the action of the operators. Even more, there is, in general, no guarantee that every polarized function can be reached from the vacuum through the action of the group. That would be true, for instance, for finite-dimensional compact semisimple groups and for Kac-Moody groups, as a consequence of the Borel-Weil-Bott theorem. A detailed discussion of that problem here associated with the Virasoro subgroup can be found in Ref. 4. In addition, instead of null vectors we find true null linear combinations of vector states and then the carrier space

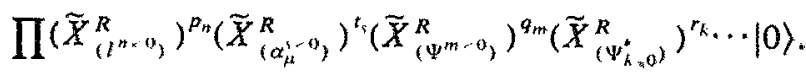

is irreducible. From (6.18) we deduce that the structure of the state $\widetilde{X}_{(i-h-0)}^{R}|0\rangle$ is identical with that of the state

$$
\begin{aligned}
& \left\{-\frac{1}{i \lambda} \sum_{m, r} f_{m-k} r \widetilde{X}_{\left(\Psi^{\prime}\right)}^{R} \widetilde{X}_{\left(\psi_{m,}^{*}\right)}^{R}\right. \\
& \left.+\frac{1}{2 i \kappa} g_{\mu v} \tilde{X}_{\left(\alpha_{v}{ }^{-k}\right.}^{R}, \widetilde{X}_{\left(\alpha_{k}^{m}\right)}^{R}\right\}|0\rangle \text {, }
\end{aligned}
$$

although only for special values of $d$ and $c^{\prime}$ they are proportional:

$$
d=26, \quad c^{\prime}=24 .
$$

In fact, the lowest orders of $(6.20)$ are

$$
\begin{aligned}
-i \lambda & \sum_{0 \leqslant m<k}(m+k)\left|\Psi_{m-k}^{*} \Psi^{m}\right\rangle+\frac{l k}{2} g^{\mu v} \sum_{0>m>-k}(-m) \\
\times & (k+m)\left|\alpha_{\mu}^{k+m} \alpha_{v}^{-m}\right\rangle+\sum_{0<m<k}(m+k)(m-2 k) \\
\times & \left|l^{k}\right\rangle-\frac{1}{2} g_{\mu v} \sum_{0>m>-k}(-m) g^{\mu v}(-k-m)\left|l^{k}\right\rangle \\
= & -\left[\left(\frac{13}{6}-\frac{d}{12}\right) k^{3}-\left(\frac{1}{6}-\frac{d}{12}\right) k\right]\left|l^{k}\right\rangle-i \lambda \\
& \times \sum_{0<m<k}(m+k)\left|\Psi_{m-k}^{*} \Psi^{m}\right\rangle \\
& +\frac{i \kappa}{2} g^{\mu v} \sum_{0>m>-k}(-m)(k+m)\left|\alpha_{\mu}^{k+m} \alpha_{v}^{-m}\right\rangle .
\end{aligned}
$$

For these values, the irreducible carrier space is made out of states created by just the operators $\widetilde{X}_{(\Psi)}^{R}, \widetilde{X}_{(\psi *)}^{R}$, and $\widetilde{X}_{(\alpha)}^{R}$ from the vacuum (similarly, the operators $\widetilde{X}_{(\pi)}^{R}, \widetilde{X}_{(T)}^{R}$, and $\tilde{X}_{(q)}^{R}$ also admit a Sugawara-like construction). The critical values thus appear as a singularity in the carrier space for the irreducible representations of the corresponding supergroup 
$\widetilde{\mathfrak{B}}\left(\operatorname{diff} S^{1}, \operatorname{diff} S^{1}\left(S \mathscr{L} \mathbb{R}^{1, d-1}\right)\right.$. These results are illustrated in Fig. 1.

According to our "non-Abelian" quantization mechanism the next step in determining the physical wave functions consists in imposing the $T$-equivariance conditions (2.19) with $\mathscr{T} \equiv \hat{\mathfrak{b}}$ (diff $S^{1}$ ) restricted to (2.20) which turns out to be

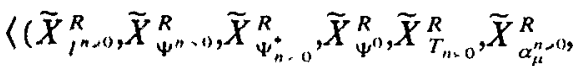

$$
\begin{aligned}
& \left.\left.\widetilde{X}_{\pi_{1}^{m-0}}^{R}\right) \cap \mathcal{T}, \Xi\right\rangle=\left\langle\widetilde{X}_{l^{n, 0}}^{R}, \widetilde{X}_{\psi^{\prime \prime}>0}^{R}, \widetilde{X}_{\psi_{n<0}^{*}}^{R}, \widetilde{X}_{\psi^{n}}^{R}, \widetilde{X}_{T_{n<0},}^{R}, \Xi\right\rangle \text {. }
\end{aligned}
$$

They are (the $\Xi$ condition was already imposed)

$$
\begin{aligned}
& \widetilde{X}_{l^{n, 0}}^{R} \Psi=i a \delta_{m, 0} \Psi, \\
& \widetilde{X}_{\psi^{n, 0}}^{R} \Psi=0, \\
& \widetilde{X}_{\psi_{n-0}^{+} \Psi}^{R} \Psi=0, \\
& \widetilde{X}_{T_{n, 0}}^{R} \Psi=0,
\end{aligned}
$$

where $a$ is a real number [integer if $L_{0}$ generate the compact $U$ (1) subgroup of diff $S^{1}$ ] characterizing the one-dimensional representation $d D$. Equation (6.23) simply says that the physical states are "proportional to the ghost vacuum $|0\rangle "$ (in the sense that there is no excited ghost state) and therefore $(6.22)$ reproduces the ordinary string constraints for the already obtained critical values $d=26, c^{\prime}=24$. In fact, the Sugawara version of the operator $\widetilde{X}_{l^{n>0}}^{R}$ [see the formula (6.20)] $\sim: g_{v \mu} \widetilde{X}_{\alpha^{m+n}}^{R} \widetilde{X}_{\alpha_{v}^{-n}}^{R}+f_{m n}^{r} \widetilde{X}_{\psi^{r}}^{R} \widetilde{X}_{\psi_{m}^{*}}^{R}:$, acting on states proportional to $|0\rangle \times$ ghosts is restricted to $: g_{v \mu} \widetilde{X}_{\alpha^{m}+n}^{R} \widetilde{X}_{\alpha_{v}^{-n}, .}^{R}$. Equation (6.24) then says nothing new since the operators $\widetilde{X}_{T_{n \vee 0}}^{R}$ are of the form $\sim \widetilde{X}_{\psi^{*}}^{R} \widetilde{X}_{\psi^{*}}^{R}$.

We must point out that the spurious states are generated by the operators (in the enveloping algebra) of the right polarization of $\mathscr{T}=\tilde{\mathrm{b}}\left(\operatorname{diff} S^{1}\right)$ containing those operators absent from the equivariance conditions (6.22-6.24). They are eliminated by means of the quotient (in the sense of vector spaces) given by

$$
\begin{aligned}
& \widetilde{X}_{q}^{R} \psi \sim 0 \text {, } \\
& \widetilde{X}_{l^{n}, 0}^{R} \psi \sim 0, \quad \widetilde{X}_{\psi^{n-0}}^{R} \psi \sim 0, \quad \widetilde{X}_{\psi_{n, 0}^{*}}^{R} \psi \sim 0, \quad \widetilde{X}_{T_{n, 0}}^{R} \psi \sim 0 .
\end{aligned}
$$

When the real parameter $a$ characterizing the quantization coincides with the critical value $c^{\prime} / 24=1$, conditions (6.22)-(6.24) are compatible with an extra condition

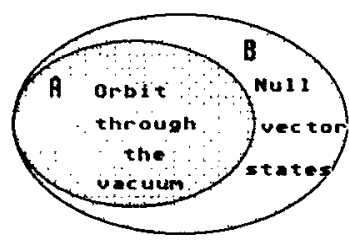

d) $d * 26,=1$.

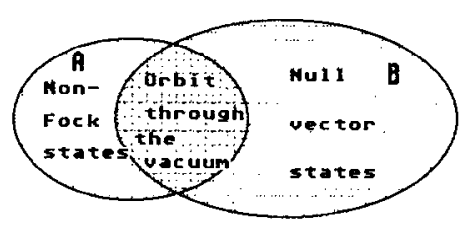

b) $d=26, a=1$.
FIG. 1. A is the set of polarized wave functions. B is the Verma module. $A \cap B$ is the irreducible Fock space. In (a) all the polarized wave functions can be obtained from the vacuum by the group action. In (b) there are nonFock states and the irreducible space is smaller.
$\widetilde{X}_{q}^{k} \psi=0(\hat{Q} \psi=0)$. Even more, this condition along with (6.23) implies $\widetilde{X}_{l^{n, 0}}^{R} \psi=\delta_{n, 0} \psi$ as a consequence of the commutation relations. The condition (6.25a) eliminates the spurious states and with the above-mentioned $\widetilde{X}_{q}^{R} \psi=0$ gives rise to the $Q$-cohomology of physical states with ghost number $-1 / 2$ [due to (6.23)]. The remainder, (6.25b), does not impose any additional condition.

We finally point out that the condition $\widetilde{X}_{q}^{R} \psi=0$, unlike in the gauge theories (see Ref. 38 where the electromagnetic field is studied in a similar way), does not appear directly in the set of equivariance conditions (6.22)-(6.24) since the $Q$ cohomology structure is associated with the critical values $(d=26, a=1)$.

To conclude we must remark that using additional BRST generators to describe a particular constrained system corresponds merely to a different choice of quantum and structure group and does not lead to essentially new features from the point of view of our general scheme. For the examples treated in the present paper, the inclusion of ghost variables is optional and only leads to a different characterization of the physical states in terms of the $Q$ cohomology. Nevertheless, it does not exclude the possibility that the search for a quantum group associated with a given constrained dynamical system leads to a BRST-like group as the only solution. That is the case for example of the free electromagnetic field where the incorporation into the group law of the gauge transformation properties of $A_{\mu}(x)$, $A_{\mu}(x) \rightarrow A_{\mu}(x)+\partial_{\mu} \Phi(x)$ requires a nonconventional bosonic generator providing some sort of BRST structure. ${ }^{38}$

However, the case of Yang-Mills theory might be quite different, since due to the non-Abelianness which introduces a nontrivial interaction among the fields of the gauge group, the use of anticommuting ghost generators may considerably simplify the search for a closing quantum group. The application of the GAQ approach to Yang-Mills theory is also expected to provide some answers to important questions concerning renormalization and anomalies, and is momentarily under investigation.

\section{ACKNOWLEDGMENTS}

We thank C. J. Isham for reading a preliminary version of this paper.

'V. Aldaya, R. Loll, and J. Navarro-Salas, Phys. Lett. B 225, 340 (1989)

${ }^{2}$ V. Aldaya and J. A. de Azcárraga, J. Math. Phys. 23, 1297 (1982); V. Aldaya and J. de Azcárraga, Ann. of Phys. (NY) 165, 484 (1985).

${ }^{3}$ V. Aldaya, J. Navarro-Salas, and A. Ramirez, Commun. Math. Phys. 121, 541 (1989).

${ }^{4}$ V. Aldaya and J. Navarro-Salas, Commun. Math. Phys. 126, 575 ( 1990).

${ }^{5}$ C. Becchi, A. Rouet, and R. Stora, Phys. I ett. B 52, 344 (1974); I. V. Tyutin, Lebedev FIAN Rep. 39 (1975) (unpublished).

${ }^{6}$ E. S. Fradkin and G. A. Vilkovisky, Phys. Lett. B 55, 224 (1975); I. A. Batalin and G. A. Vilkovisky, Phys. Lett. B 69, 309 (1977).

${ }^{7}$ D. McMullan, J. Math. Phys. 28, 428 (1987).

${ }^{8}$ R. Loll, Commun, Math. Phys. 119, 509 (1988).

${ }^{9}$ B. Kostant and S. Sternberg, Ainl. of Phys. (NY) 176, 49 (1987).

${ }^{10}$ M. J. Bowick and S. G. Rajeev, Phys. Rev. Lett. 58, 535 (1987); CTP XX, 1494 (1987).

${ }^{11}$ M. Kato and K. Ogawa, Nucl. Phys. B 212, 443 (1983); S. Hwang, Phys. Rev. D 28, 2614 (1983). 
${ }^{12}$ E. Witten, Commun. Math. 114, 1 (1988); P. Goddard and D. Olive, Int. J. Mod. Phys. A 1, 303 (1986).

${ }^{13}$ J. M. Souriau, Structure des Systemes Dynamiques (Dunod, Paris, 1970); B. Kostant, Quantization and Unitary Representation, in Lecture Notes in Mathematics Vol. 179 (Springer-Verlag, Berlin 1970); A. A. Kirillov, Elements of the Theory of Representations (Springer-Verlag, Berlin 1975).

${ }^{14} \mathrm{C}$. J. Isham, in Relativity, Groups and Topology II, Proceedings of the 1983 Les Houches Summer School edited by B. DeWitt and R. Stara (North-Holland, Amsterdam, 1984); C. J. Isham and N. Linden, Class. Quant. Gravit. 5, 71 (1988).

${ }^{15}$ J. Navarro-Salas and J. R. Klauder, Class, Quan. Gravit. (to appear).

${ }^{16} \mathrm{~V}$. Aldaya and J. Navarro-Salas, Lett. Math. Phys. 16, 117 (1988).

${ }^{17}$ R. Hermann, Vector Bundles in Mathematical Physics, (Benjamin, New York, 1970), Vols. I and II.

${ }^{18}$ F. Bayen, M. Flato, C. Fronsdal, A. Lichnerowicz, and D. Sternheiner, "Deformation Theory and Quantization. I: Deformation of Symplectic Structures," Ann. Phys. 111, 61 (1978); "Deformation Theory and Quantization. II: Physical Applications," Ann. Phys. 111, 111 (1978).

${ }^{19}$ V. G. Drinfel'd, Soviet Math. Dokl. 27, 68 (1983);32, 254 (1985); Quantum Groups, Proceeding of the International Congress of Mathematicians, Berkely, CA. (Publisher, City, 1986), p. 798; M. E. Sweedler, Hopf Algebras. Mathematical Lecture Note Series (Benjamin, New York, 1969).

${ }^{20} \mathrm{C}$. Godbillon, Géométrie Differentielle et Mécanique Analytique (Hermann, Paris, 1969); R. Abraham and J. E. Marsden, Foundations of Mechanics (Benjamin, New York, 1978).

${ }^{21}$ V. Aldaya and J. A. de Azcárraga, Fortschr. Phys. 35, 437 (1987).

${ }^{22}$ V. Aldaya and J. Navarro-Salas, Commun. Math. Phys. 113, 375 (1987).

${ }^{27}$ S. Kobayashi and K. Nomizu, Foundations of Differential Geometric (Interscience, New York, 1963), Vols. I and II.

${ }^{24}$ For instance, in string theory a full polarization should be made out of the Virasoro generators as well as "half the loop space generators" $\alpha^{\prime \prime}$, but it does not close (see later on in Sec. VI B).
${ }^{2 s} \mathrm{~V}$. Aldaya and J. A. de Azcárraga, in Modern Developments in Analytical Mechanics, edited by S. Benenti, M. Francaviglia and A. Lichnerowicz, Acta Acad. Sci. Taurinensis 177, Suppl. 1 (1983).

${ }^{26} \mathrm{C}$. Itzykson and J. B. Zuber, Quantum Field Theory (McGraw-Hill, New York, 1985).

${ }^{27}$ L. Bonora and P. Cotta-Ramusino, Commun. Math. Phys. 87, 589 (1983).

${ }^{28}$ M. Henneaux, Phys. Rep. 126, 1 (1985)

${ }^{29} \mathrm{~J}$. D. Stasheff, "Constrained Poisson Algebras and Strong Homotopy Representations," preprint Univ. of North Carolina, (1987).

${ }^{30} \mathrm{~J}$. Fisch, M. Henneaux, J. Stasheff, and C. Teitelboim, Commun. Math. Phys. 120, 379 (1989).

${ }^{31}$ J. P. Serre, Lie Algebras and Lie Groups (Benjamin, New York, 1965).

${ }^{32}$ M. J. Bowick and F. Gursey, Phys. Lett. B 175, 182 (1986).

${ }^{33}$ T. Kawai, Phys. Lett. B 168, 355 (1986).

${ }^{34}$ H. P. Jacobsen and V. Kac, Lecture Notes in Physics, Vol. 226, edited by N. Sanchez (Springer, New York, 1985), pp. 1-20.

${ }^{35}$ P. Fayet and S. Ferrara, Phys. Rep. C 32, 249 ( 1977 ).

${ }^{36}$ In the study of the representations of the subgroup diff $S^{\prime}$ the vacuum is replaced with the "semi-infinite wedge product" $\Pi_{n<0} \Psi^{n}$ and then the $\widehat{L}_{0}$ is regularized by means of the definition $2 n L \equiv\left[L_{n}, L_{-n}\right]$ but for an anomaly which is now present [see I. B. Frenkel, H. Garland, and G. $]$. Zuckerman, Proc. Natl. Acad. Sci. (USA) 83, 8442 (1986)].

${ }^{37}$ In writing down the fields $(6.11)$ and $(6.13)$ we have interchanged what would have been $g^{\prime}$ and $g$ in the group law of $\widetilde{\mathcal{B}}$ (diff $S^{1}$; diff $S \mathcal{O} \mathscr{Y}^{1, d-1}$ ) had we written it explicitly. Therefore, if we want to see these fields as an enlargement of those corresponding to $\widehat{\mathcal{B}}$ (diff $S^{\prime}$ ) we must compare (6.11) with $(4.6)$ and $(6.13)$ with $(4.5)$. The reason for that change is just a matter of mere convenience; the matrices $R, R^{-1}$, and $U$ in most left generators do not factor out in the polarization conditions to be imposed, since not all the field subscripts $n \in \mathbb{Z}$ are present in these equations.

${ }^{38}$ V. Aldaya, R. Loll, and J. Navarro-Salas, Imperial TP/88-89/19 (Phys. Lett. $B$ to appear). 
Journal of Mathematical Physics is copyrighted by the American Institute of Physics (AIP). Redistribution of journal material is subject to the AIP online journal license and/or AIP copyright. For more information, see http://ojps.aip.org/jmp/jmpcr.jsp Copyright of Journal of Mathematical Physics is the property of American Institute of Physics and its content may not be copied or emailed to multiple sites or posted to a listserv without the copyright holder's express written permission. However, users may print, download, or email articles for individual use. 